\title{
Pricing Two Trees when Mildew Infests the Orchard: How Does Contagion Affect General Equilibrium Asset Prices?
}

\author{
Nicole Branger* \\ Holger Kraft** \\ Christoph Meinerding***
}

This version: March 11, 2011

\begin{abstract}
This paper analyzes the equilibrium pricing implications of contagion risk in a twotree Lucas economy with CRRA preferences. The dividends of both trees are subject to downward jumps. Some of these jumps are contagious and increase the risk of subsequent jumps in both trees for some time interval. We show that contagion risk leads to large price-dividend ratios for small assets, a joint movement of prices in the case of a regime change from the calm to the contagion state, significantly positive correlations between assets, and large positive betas for small assets. Whereas disparities between the assets with respect to their propensity to trigger contagion barely matter for pricing, the prices of robust assets that are hardly affected by contagion and excitable assets that are severely hit by contagion differ significantly. Both in absolute terms and relatively to the market, the price of a small safe haven increases if the economy reaches the contagion state. On the contrary, the price of a small, contagion-sensitive asset exhibits a pronounced downward jump.
\end{abstract}

Keywords: Contagion, General Equilibrium, Asset Pricing

JEL: G01, G12

${ }^{*}$ Finance Center Münster, Westfälische Wilhelms-Universität Münster, Universitätsstr. 14-16, 48143 Münster, Germany. E-mail: nicole.branger@wiwi.uni-muenster.de.

**Department of Finance, Goethe University, 60323 Frankfurt am Main, Germany. E-mail: holgerkraft@finance.uni-frankfurt.de.

***Finance Center Münster, Westfälische Wilhelms-Universität Münster, Universitätsstr. 14-16, 48143 Münster, Germany. E-mail: christoph.meinerding@wiwi.uni-muenster.de.

The paper was presented at the 17th Annual Meeting of the German Finance Association in Hamburg, the 14th Conference of the Swiss Society for Financial Market Research, and at research seminars at the universities of Bielefeld and Muenster. The authors would like to thank the conference and seminar participants and discussants for many helpful comments and suggestions. Holger Kraft gratefully acknowledges financial support by Deutsche Forschungsgemeinschaft (DFG). 


\title{
1 Introduction and Motivation
}

The concept of financial contagion describes a situation where losses in one company, one sector of the economy or one country significantly increase the risk of subsequent losses both in the same and in other companies, sectors or countries. However, although a vast amount of literature deals with the various aspects of contagion, the preface of the insightful anthology of Claessens and Forbes (2001) still applies:

\begin{abstract}
'No sooner had the Asian crisis broken out in 1997 than the witch-hunt started.
With great indignation every Asian economy pointed fingers. They were innocent bystanders. The fundamental reason for the crisis was this or that most prominently contagion - but also the decline in exports of the new commodities (high-tech goods), the steep rise of the dollar, speculators, etc. The prominent question, of course, is whether contagion could really have been the key factor and, if so, what are the channels and mechanisms through which it operated in such a powerful manner. The question is obvious because until 1997, Asia's economies were generally believed to be immensely successful, stable and well-managed.'
\end{abstract}

A crucial issue in modeling financial contagion is its time dimension. ${ }^{1}$ Contagion is not an event that takes place at a single point in time. Instead, the risky 'contagious state' prevails for some time interval before the economy eventually reverts to the initial 'calm state'. Consequently, contagion affects the investment opportunities for some time period. The question then is how contagion affects the equilibrium in the economy. How is the threat of contagion reflected in prices today, and how do prices change if contagion hits the economy? Do we observe differential pricing in the cross-section if assets differ in their propensity to trigger contagion or in their degree of robustness against contagion risk?

In this paper, we analyze the impact of contagion risk on asset prices and asset price dynamics in a general equilibrium. We rely on a Lucas-tree economy with several trees which was first analyzed in Cochrane, Longstaff, and Santa-Clara (2008), who consider two trees and a log investor, and Martin (2009), who studies a Lucas orchard with several trees and assumes general CRRA preferences. The representative investor in our economy also has CRRA preferences. Dividends are subject to diffusion risk and to downward jumps. The jump intensities follow a two-state Markov chain: they are moderate in the

\footnotetext{
${ }^{1}$ See, e.g., Ait-Sahalia, Cacho-Diaz, and Laeven (2010)
} 
'calm state', but increase significantly as soon as the economy enters the 'contagion state'. A distinctive feature of our model is that jumps from the calm state to the contagion state are triggered by (or happen simultaneously with) a drop in the dividends of either of the two trees. In a sense, financial contagion can be viewed as an extremely virulent disease among the trees (one might, e.g., think of the classical mildew).

Our paper makes three main contributions to the literature. First, we derive a semi-closed form solution for the price-dividend ratio (and thus all other key asset pricing figures) in our model. Second, we analyze the impact the threat of contagion has on prices and price dynamics, and we compare prices in the calm state and in the contagion state. Third, we analyze the cross-sectional effects of contagion, i.e. the differential pricing of assets with respect to their exposure to contagion risk.

In more detail, our contributions are as follows. For calculating the price-dividend ratios, we start from the solution of Martin (2009) for the case of iid dividends in a Lucas orchard and follow Branger, Schlag, and Wu (2011) in extending it to the case of non-iid dividends. A key quantity is the characteristic function of future dividends. We show that our model is affine so that the characteristic function has a standard form (see Duffie, Pan, and Singleton (2000)). Furthermore, we manage to simplify the resulting formula for the price-dividend ratio significantly and are able to reduce the number of numerical integrations to one like in the case of iid dividends.

Second, we study the impact of contagion risk on equilibrium asset prices. We start with the case where both trees have identical parameters. As shown by Cochrane, Longstaff, and Santa-Clara (2008) and Martin (2009), there are spill-over effects between the trees. The asset prices depend on the dividends of both trees and not just on the own dividends since the stochastic discount factor is driven by the joint behavior of both dividends. These spill-over effects lead to large price-dividend ratios for small trees, excess volatilities of large trees, positive correlations between the prices even if dividends are uncorrelated, and nonzero exposures of prices to shocks in the dividends of the other asset.

In our model, regime changes from the calm to the contagion state and vice versa add a further risk dimension. Since price-dividend ratios depend on the current regime, jumps into the contagion state lead to simultaneous large price changes in both assets, and jumps back into the calm state, of course, to a reverse change. These simultaneous large price jumps increase the already positive correlations between the assets. In particular, these correlations remain significant even when one asset becomes small (and its dividend no longer contributes to systematic consumption risk). Our model thus endogenously 
generates additional systematic risk and thus an additional beta for small assets. This effect is particularly pronounced in the contagion state.

Third, we analyze the differences in prices and price dynamics that are caused by differences in the characteristics of the dividends. This allows us to assess the impact of contagion risk on the cross-sectional pricing of stocks. We find that the propensity to induce contagion, i.e. the question of which of the assets really triggers contagion, barely matters for pricing. There are basically no differences between the prices of assets that induce contagion and assets that never do so.

On the contrary, the consequences of contagion are of first-order importance. We show that there are indeed significant differences between the prices of robust assets (whose jump intensity is only slightly higher in the contagion state) and excitable assets (which are heavily affected by contagion in the sense that the intensities of downward jumps in their dividends increase strongly). The price-dividend ratio of a small robust asset is much greater than the price-dividend ratio of an equally small excitable asset. While there is always a large excess demand for a small asset due to diversification needs of the investor (see Cochrane, Longstaff, and Santa-Clara (2008)), we find additional demand for the small safe haven which drives its price up even further. The price-dividend ratio of a small excitable asset, on the other hand, is hardly greater than that of the market since the diversification benefits are nearly offset by the less attractive growth prospects.

The different characteristics of the assets also lead to different price changes upon entering the contagion state. The excess demand for the safe haven represented by the small robust asset in the contagion state drives its price up both in absolute terms as well as relatively to the market (flight to quality). The price of a small excitable asset drops relatively to the market and, depending on the parameters, also in absolute terms. The reason is that the jump intensity of the large (robust) asset increases only mildly, and, thus, the small asset takes the whole burden of the economic downturn.

Moreover, the mere fact that contagion may happen at some future point in time increases the systematic risk in the economy: even small assets whose dividends are nearly independent of overall consumption have positive market betas that can be as high as 0.8 in the contagion state in our example. The risk of joint price jumps (not joint dividend jumps) due to a regime change increases the systematic risk in the economy significantly. Finally, volatilities are larger in the contagion state than in the calm state due to the increase in the overall level of risk in this state. Volatilities are thus anti-cyclical in our model and increase if the economic conditions worsen. 
Our paper is related to several strands of the literature. First of all, we build on the growing research on asset pricing with multiple trees. Cochrane, Longstaff, and Santa-Clara (2008) analyze the equilibrium in a Lucas tree economy with two structurally identical trees with iid dividends, diffusion risk only, and a log investor. Martin (2009) extends this setup to several trees, jump-diffusion processes, and CRRA utility. Branger, Schlag, and Wu (2011) consider a setup where the dividend growth rate is stochastic for one of the two trees so that dividends are no longer iid and analyze the implications of learning. Formally, the latter two approaches rely on an application of the generalized transform analysis of Chen and Joslin (2010). Buraschi, Porchia, and Trojani (2010) also consider a Lucas orchard with jump risk and two states for jump intensities. They include more than two trees and focus on the implications of learning about the true state. In their model, however, disasters only have a temporary effect since each tree can either be in the recovery state (with high dividends) or in the recession state (with low dividends). This implies a kind of mean-reversion for the dividends. In contrast, we focus on the implications of persistent jumps which reduce the level of dividends in all future periods.

There is also a huge literature on the impact of jumps onto asset prices. Naik and Lee (1990) derive the equilibrium in a one-tree economy with utility from terminal wealth where the dividend follows a jump-diffusion process. Rietz (1988) and Barro (2006, 2009) show that rare but severe disasters help to explain the equity premium puzzle. Wachter (2010) analyzes the impact of a time-varying (exogenous) jump intensity on the variance of returns in a model with Epstein-Zin preferences. Gabaix (2010) focuses on time-varying jump intensities and time-varying recovery rates in a model with CRRA utility in order to solve some famous asset pricing puzzles. Backus, Chernov, and Martin (2011) draw a link between consumption-based asset pricing models and standard option pricing theory. They find that option prices imply more frequent, but also more modest jumps than the disaster literature in asset pricing assumes.

Another strand of related literature deals with the correct notion of contagion, its econometric detection and its financial implications. The book of Claessens and Forbes (2001) provides a detailed synopsis of the different terms which are around in this literature. Longin and Solnik (2001) as well as Forbes and Rigobon (2002), among others, analyze the time-varying behavior of stock return correlations. Differing from this type of studies, our paper explicitly focuses on jump risk as the prevalent source of contagion. Bae, Karolyi, and Stulz (2003) evaluate contagion via the coincidence of extreme return shocks of stock indices across several countries all around the globe. They find significant evidence for the existence of contagion. Our paper differs from this kind of studies in that we explicitly 
focus on the time dimension of contagion. Instead of large simultaneous dividend movements, our model involves dividend shocks that increase all other loss probabilities for a certain time period and, thus, have a longer-lasting impact.

Ait-Sahalia, Cacho-Diaz, and Laeven (2010) use a definition of financial contagion that is closest to ours. They focus on developing and implementing an estimation procedure for a model akin to ours which captures the empirically observed jump clustering. In their setup, economic shocks propagate around the globe within days whereas, in our benchmark parametrization, contagion lasts for several months. However, as our numerical results show, the duration of the contagion state is of second-order importance only. The mere fact that contagion is modeled as an economic state instead of a single disastrous event drives most of our findings.

The remainder of this paper is structured as follows. Section 2 explains the model setup. The equilibrium pricing relations and the results for the case of structurally identical trees are presented in Section 3. Section 4 analyzes the cross-sectional pricing implications of contagion risk. Some robustness checks are performed in Section 5. Section 6 concludes.

\section{Model Setup}

\subsection{Dividend Dynamics}

We consider a continuous-time Lucas tree economy with an infinite horizon. There are two trees producing the same perishable consumption good which serves as numeraire. The dividends of the trees follow a jump-diffusion process.

We capture contagion risk by a Markov chain the state of which influences the jump intensities. The economy can be in either of two states, the calm or contagion one. We introduce a state variable $p_{t}$ which can be viewed as the indicator variable of the Markov chain: the economy is in the calm state if $p_{t}=1$ and in the contagion state if $p_{t}=0$.

The trees $A$ and $B$ produce the dividends $D_{A}$ and $D_{B}$. The dynamics of the log dividends $y_{A}=\ln D_{A}$ and $y_{B}=\ln D_{B}$ and of the state variable $p_{t}$ are

$$
\begin{aligned}
d y_{A, t} & =\mu_{A}\left(p_{t}\right) d t+\sigma_{A} d W_{t}^{A}+\log \left(1+L_{A}\right) \sum_{j=1,2,3} d N_{t}^{A, j} \\
d y_{B, t} & =\mu_{B}\left(p_{t}\right) d t+\sigma_{B} d W_{t}^{B}+\log \left(1+L_{B}\right) \sum_{j=1,2,3} d N_{t}^{B, j} \\
d p_{t} & =-\left(d N_{t}^{A, 2}+d N_{t}^{B, 2}\right)+d N_{t}^{4} .
\end{aligned}
$$


The drifts of the dividends $\mu_{A}$ and $\mu_{B}$ are state-dependent, and we set $\mu_{i}\left(p_{t}\right)=p_{t} \mu_{i}^{\text {calm }}+$ $\left(1-p_{t}\right) \mu_{i}^{\text {cont }}$. The Wiener processes $W^{A}$ and $W^{B}$ are correlated with correlation $\rho$, and we assume that the diffusion volatilities are constant. Furthermore, the dividends can jump with a relative jump size of $L_{i}<0$, which is constant in our model. ${ }^{2}$ The jumps are driven by the Poisson processes $N$.

We now first give the economic description of the jump structure in our model before we turn to how it is captured formally. In the calm state $\left(p_{t}=1\right)$, two types of jumps in each asset can occur: First there can be jumps that do not change the state. These jumps have an intensity of $\lambda_{i}^{\text {calm,calm }}$. The second type of jumps does not only decrease the dividend, but also triggers a regime change from 'calm' to 'contagion'. The intensity of these jumps is denoted by $\lambda_{i}^{\text {calm,cont }}{ }^{3}$ Put together, the total jump intensity for dividend $i$ in the calm state is $\lambda_{i}^{\text {calm,calm }}+\lambda_{i}^{\text {calm,cont }}$. In the contagion state $\left(p_{t}=0\right)$, the intensity for a downward jump in dividend $i$ is $\lambda_{i}^{\text {cont,cont }}$, which is assumed to be larger than the jump intensity in the calm state. Furthermore, this type of jumps does not change the state, i.e. the economy stays in the contagion state. Finally, the economy can also jump back to the calm state with intensity $\lambda^{\text {cont,calm }}$. Such a jump does not trigger losses in any of the dividends.

Formally, to model all possible jumps, we introduce the Poisson processes $N^{i, j}(i=$ $A, B, j=1,2,3)$ and $N^{4}$ with intensities $\lambda_{t}^{i, j}$ and $\lambda_{t}^{4}$, respectively. $N^{i, 1}$ and $N^{i, 2}$ are jumps that can happen in the calm state only. We set $\lambda_{t}^{i, 1}=p_{t} \lambda_{i}^{\text {calm,calm }}$, i.e. $j=1$ corresponds to a jump that does not change the state, and $\lambda_{t}^{i, 2}=p_{t} \lambda_{i}^{\text {calm,cont }}$, i.e. $j=2$ corresponds to a jump from the calm to the contagion state. The multiplication with $p_{t}$ ensures that the jump intensities are indeed zero in the contagion state (where $p_{t}=0$ ). Similarly, the intensities of $N^{i, 3}$ and $N^{4}$ are $\lambda_{t}^{i, 3}=\left(1-p_{t}\right) \lambda_{i}^{\text {cont,cont }}$, i.e. $j=3$ captures jumps in the contagion state, and $\lambda_{t}^{4}=\left(1-p_{t}\right) \lambda^{\text {cont,calm }}$, describing jumps back into the calm state. Again, this ensures that the corresponding jumps can only occur in the contagion state.

To summarize, the dividends follow RCLL processes. The drifts, the variance-covariance matrix of the diffusion components, and the jump intensities are affine functions of the state variable $p_{t}$. The model is thus affine, and there are a closed-form solutions for the characteristic functions of the log-dividends (see, e.g., Duffie, Pan, and Singleton (2000)).

\footnotetext{
${ }^{2}$ We could also allow for a stochastic jump size. However, this would complicate the model and notation without adding to our main results.

${ }^{3}$ In the following, we will call the asset whose dividends exhibit this second kind of jumps the 'contagion-inducing' asset.
} 


\subsection{Representative Investor}

Our economy is populated by a representative investor with CRRA utility and timeadditive preferences. His subjective time preference rate is denoted by $\delta$, and his relative risk aversion is $\gamma$. Under the assumption of an infinite planning horizon, his expected utility is

$$
E\left[\int_{0}^{\infty} e^{-\delta t} \frac{C_{t}^{1-\gamma}}{1-\gamma} d t\right]
$$

In the following, we assume $\gamma>1$. Thus, the intertemporal elasticity of substitution is $1 / \gamma<1$

\subsection{Dynamics of Aggregate Consumption and Dividend Share}

In equilibrium, aggregate consumption equals aggregate dividends. The dynamics of consumption $D_{m, t}=D_{A, t}+D_{B, t}$ are given by

$$
\begin{aligned}
\frac{d D_{m t}}{D_{m, t-}}= & {\left[s_{t}\left(\mu_{A}+0.5 \sigma_{A}^{2}\right)+\left(1-s_{t}\right)\left(\mu_{B}+0.5 \sigma_{B}^{2}\right)\right] d t+s_{t} \sigma_{A} d W_{t}^{A}+\left(1-s_{t}\right) \sigma_{B} d W_{t}^{B} } \\
& +s_{t} L_{A} \sum_{j=1,2,3} d N_{t}^{A, j}+\left(1-s_{t}\right) L_{B} \sum_{j=1,2,3} d N_{t}^{B, j}
\end{aligned}
$$

where $s_{t}$ denotes the dividend share of asset A at time $t$ :

$$
s_{t}=\frac{D_{A, t}}{D_{A, t}+D_{B, t}} .
$$

In the following, the term 'size' is used as a synonym for the dividend share of a tree. E.g., asset A is called 'large' if $s_{t}$ is near 1 . The dynamics of $s_{t}$ follow from Ito's Lemma:

$$
\begin{aligned}
d s_{t}=s_{t}\left(1-s_{t}\right)[ & {\left[\mu_{A}-\mu_{B}-\left(s_{t}-0.5\right)\left(\sigma_{A}^{2}+\sigma_{B}^{2}-2 \rho \sigma_{A} \sigma_{B}\right)\right] d t+\sigma_{A} d W_{t}^{A}-\sigma_{B} d W_{t}^{B} } \\
& \left.+\sum_{j=1,2,3} \frac{L_{A}}{1+L_{A} s_{t}} d N_{t}^{A, j}-\sum_{j=1,2,3} \frac{L_{B}}{1+L_{B}\left(1-s_{t}\right)} d N_{t}^{B, j}\right] .
\end{aligned}
$$

Downward jumps in dividend A reduce the dividend share of asset A, whereas downward jumps in $\mathrm{B}$ increase it. In the case of identical trees, the (absolute) sensitivity of $s_{t}$ to shocks in one of the dividends is largest if the trees have equal size. It converges to zero if one of the trees dominates the economy. 
The local variance of consumption equals

$$
\begin{aligned}
\operatorname{Var}_{t}\left[\frac{d D_{m t}}{D_{m, t-}}\right]= & s_{t}^{2}\left(\sigma_{A}^{2}+L_{A}^{2} \sum_{j=1,2,3} \lambda_{t}^{A, j}\right) d t+\left(1-s_{t}\right)^{2}\left(\sigma_{B}^{2}+L_{B}^{2} \sum_{j=1,2,3} \lambda_{t}^{B, j}\right) d t \\
& +2 s_{t}\left(1-s_{t}\right) \rho \sigma_{A} \sigma_{B} d t .
\end{aligned}
$$

It depends on the dividend share $s_{t}$ and on the state variable $p_{t}$ (via the jump intensities) and is thus stochastic. In the case of identical trees, it is the smallest if both trees have the same size.

\subsection{Parametrization}

For our numerical examples, we set the subjective time discount rate of the representative investor equal to $\delta=0.03$ and his relative risk aversion equal to $\gamma=4$. Both choices are

in line with the literature. The intertemporal elasticity of substitution is thus given by $\gamma^{-1}=0.25$, which is well below one. Consequently, the investor is very eager to smooth consumption over time, and the income effect dominates the intertemporal substitution effect, so that the wealth-consumption ratio is greater in bad states than in good states. Our key contributions are, however, not affected by this assumption.

Table 1 provides the parameters for the dividend dynamics in the benchmark case. In our setting, the two trees may differ along two dimensions: size and parametrization. In the following, we assume that the trees have identical parameters and call this situation the case with 'identical trees' (even if the trees have different sizes). The case with different parameters for the two trees will be analyzed in Section 4, where we study the impact of contagion on the differential pricing of the stocks in more detail.

For the dividend dynamics, we start from the diffusion case in Martin (2009). He assumes a volatility of $10 \%$ and a dividend growth rate of $2 \%$. Our model includes jumps, so that we choose a lower diffusion volatility of $8 \%$ and a greater drift conditional on no jumps of $3.5 \%$. Note that we do not model consumption and dividends separately, but assume that aggregate dividends are equal to consumption.

Jumps are assumed to happen quite frequently and are rather moderate, different from the literature on disaster risk (see, e.g., Barro $(2006,2009)$ ). Upon a jump, the corresponding dividend drops by $2 \%$. In the calm state, the intensity of a jump is 0.4 for each of the two stocks. Every other of these jumps induces contagion, i.e. triggers a change in the economic state. In the contagion state, jump intensities are five times as high as in the 
calm state. Finally, the intensity for a jump from the contagion to the calm state is 2 , so that the economy spends on average 6 months in the contagion state. The stationary probabilities of the calm and the contagion state are 5/6 and $1 / 6$, respectively.

Our parametrization implies the following moments of dividend and consumption growth. In the calm state, the expected dividend growth rate is $3 \%$, which drops to $0 \%$ in the contagion state. The volatility increases from $8.1 \%$ in the calm state to $8.5 \%$ in the contagion state. The expected consumption growth equals the expected dividend growth, while the volatility of consumption depends on the dividend share. It is the largest if one of the two trees dominates and drops to $6 \%$ if both trees have equal size.

We set $\mu_{i}^{\text {calm }}=\mu_{i}^{\text {cont }}$ and thus assume that the growth rate of the trees conditional on no jumps is constant and does not depend on the state. In a production economy, one could think of the growth rate as the overall (constant) expected profitability of the production technology. Jumps are adverse events that permanently reduce the capital stock and thus also future dividends. Examples include natural disasters, the failure of a newly developed medicine, problems in obtaining a loan, the bankruptcy of a major customer, or the loss in a major lawsuit. In the contagion state, the intensity for such disasters is significantly greater.

Alternatively, one can choose the drift in the calm and contagion state such that the expected growth rates of dividends are equal in both states. In this case, the higher intensity of disasters in the contagion state is - at least in terms of expected growth offset by a higher productivity of the trees conditional on no disasters. The main effect of the contagion state is then the higher variance of dividends due to the larger jump intensities.

Our results definitely depend on which setup we choose. The first one where a larger probability of adverse events is not offset by a higher productivity might seems more appropriate to capture contagion effects. However, Section 5 provides a robustness check where we consider the case of equal growth rates in both states.

\section{Equilibrium}

In equilibrium, aggregate consumption $C$ equals aggregate dividends, $C_{t}=D_{A, t}+D_{B, t}$. The pricing kernel $\xi$ is thus given by

$$
\xi_{t}=e^{-\delta t}\left(\frac{D_{A, t}+D_{B, t}}{D_{A, 0}+D_{B, 0}}\right)^{-\gamma} .
$$


From the pricing kernel, we can derive the risk-free rate, the market prices of risk and the price-dividend ratios of the assets.

\subsection{Risk-free Rate}

The risk-free rate follows from the (negative) expected growth rate of the pricing kernel.

Proposition 1 The risk-free rate is given by

$$
\begin{aligned}
r_{f, t}= & \delta+\gamma s_{t}\left(\mu_{A}+0.5 \sigma_{A}^{2}+\sum_{j=1,2,3} L_{A} \lambda^{A, j}\right)+\gamma\left(1-s_{t}\right)\left(\mu_{B}+0.5 \sigma_{B}^{2}+\sum_{j=1,2,3} L_{B} \lambda^{B, j}\right) \\
& -0.5 \gamma(1+\gamma)\left(s_{t}^{2} \sigma_{A}^{2}+\left(1-s_{t}\right)^{2} \sigma_{B}^{2}+2 s_{t}\left(1-s_{t}\right) \rho \sigma_{A} \sigma_{B}\right) \\
& -\sum_{j=1,2,3}\left[\left(1+L_{A} s_{t}\right)^{-\gamma}-\left(1-\gamma L_{A} s_{t}\right)\right] \lambda^{A, j} \\
& -\sum_{j=1,2,3}\left[\left(1+L_{B}\left(1-s_{t}\right)\right)^{-\gamma}-\left(1-\gamma L_{B}\left(1-s_{t}\right)\right)\right] \lambda^{B, j}
\end{aligned}
$$

A proof can be found in Appendix A.

The risk-free rate has the standard form which is well known for a CRRA economy. It increases in the subjective time preference rate $\delta$ and in the expected consumption growth where the latter enters with a factor $\gamma$ (which is the inverse of the intertemporal elasticity of substitution here). The remaining terms are negative and capture the impact of precautionary savings due to diffusive volatility (second line) and jump risk (third and fourth line).

The lower panel of Figure 1 depicts the risk-free rate as well as its components in the calm state (left graph) and in the contagion state (right graph). With identical trees, the risk-free rate is the largest if both trees have equal size since the variance of consumption and thus the precautionary savings term is smallest.

The risk-free rate is much lower in the contagion state than in the calm state for two reasons. First, the expected dividend growth rate is smaller in the contagion state than in the calm state. Second, the variance of consumption is larger in the contagion state than in the calm state, and the additional precautionary savings demand lowers the risk-free rate. In our benchmark case, the first effect is the larger one. 


\subsection{Market Prices of Risk}

The market prices of risk can also be derived from the dynamics of the pricing kernel.

Proposition 2 The market price of risk for $W_{t}^{A}$ is $\gamma s_{t} \sigma_{A}$, whereas the market price of risk for $W_{t}^{B}$ is $\gamma\left(1-s_{t}\right) \sigma_{B}$. The risk premium earned on an exposure $\eta$ to jumps which induce a loss in one of the dividends $(j=1,2,3)$ is $\eta\left[1-\left(1+L_{A} s_{t}\right)^{-\gamma}\right] \lambda^{A, j}$ for jumps in dividend $A$ and $\eta\left[1-\left(1+L_{B}\left(1-s_{t}\right)\right)^{-\gamma}\right] \lambda^{B, j}$ for jumps in dividend $B$. Jumps from the contagion state back to the calm state are not priced.

The proof is given in Appendix A.

The market prices of risk have the standard form for CRRA preferences. They depend on the relative risk aversion $\gamma$ and on the exposure of aggregate consumption to the risk factors. Therefore, the market prices of risk increase in the dividend share of the corresponding tree.

The risk premia for diffusion risk are identical in the calm and in the contagion state since the exposure to diffusion risk in the dividends does not depend on the state. For jumps that induce a loss in one of the dividends, the risk-neutral jump intensities are $\left(1+L_{A} s_{t}\right)^{-\gamma} \lambda^{A, j}$ and $\left(1+L_{B}\left(1-s_{t}\right)\right)^{-\gamma} \lambda^{B, j}, j=1,2,3$, respectively. They are greater than the jump intensities under the physical measure. Consequently, the investor earns a positive risk premium on an asset that loses value upon such a jump.

Finally, notice that the premium earned on a given jump risk exposure is proportional to the intensity of the jump, i.e. to the average number of jumps per year. The investor thus earns a larger jump risk premium in the contagion state than in the calm state. However, the risk premium per unit of jump risk does not depend on the state of the economy. Consequently, there is no additional risk premium for being in the (worse) contagion state. With time-additive preferences and CRRA, state variables which only affect future consumption dynamics but not current consumption are not priced. For the same reason, jumps from the contagion state to the calm state do not carry a risk premium.

\subsection{Price-Dividend Ratios}

Given the pricing kernel (1), we can price all future payment streams. Here, we focus on pricing the market, i.e. the claim to aggregate consumption, and the two trees. For notational convenience, we denote the dividend streams of both trees by $D_{A}^{\alpha_{1}} D_{B}^{\alpha_{2}}$, where 
$\left(\alpha_{1}, \alpha_{2}\right)=(1,0)$ gives tree $A$ and $\left(\alpha_{1}, \alpha_{2}\right)=(0,1)$ gives tree $B$. The price of the market is simply the sum of the prices of asset $\mathrm{A}$ and asset $\mathrm{B}$.

Proposition 3 The price-dividend ratio of an asset with dividend stream $D_{A}^{\alpha_{1}} D_{B}^{\alpha_{2}}$ is

$$
\frac{P_{t}}{D_{A, t}^{\alpha_{1}} D_{B, t}^{\alpha_{2}}}=\int_{-\infty}^{\infty} I\left(s_{t}, v\right) J\left(v, p_{t}, \alpha_{1}, \alpha_{2}\right) d v
$$

where the functions $I$ and $J$ are given by

$$
\begin{aligned}
I(s, v) & =[s(1-s)]^{-\frac{\gamma}{2}}\left(\frac{1-s}{s}\right)^{i v}=\left(D_{A}+D_{B}\right)^{\gamma} D_{A}^{-\frac{\gamma}{2}-i v} D_{B}^{-\frac{\gamma}{2}+i v} \\
J\left(v, p, \alpha_{1}, \alpha_{2}\right) & =\frac{2 F(v)}{1+M_{2}(\theta, p)}\left(\frac{1}{M_{1}(\theta)+Z(\theta)-2 \delta}+\frac{M_{2}(\theta, p)}{M_{1}(\theta)-Z(\theta)-2 \delta}\right)
\end{aligned}
$$

and where $\theta\left(v, \alpha_{1}, \alpha_{2}\right)=\left(\alpha_{1}-\frac{\gamma}{2}-i v, \alpha_{2}-\frac{\gamma}{2}+i v, 0\right)$. The functions $F, M_{1}, M_{2}$, and $Z$ can be found in Appendix $B$.

The proof is given in Appendix B.

The price-dividend ratio of the market portfolio with dividend $D_{A}+D_{B}$ is a weighted average of the individual price-dividend ratios:

$$
\frac{P_{m, t}}{D_{m, t}}=\frac{P_{A, t}+P_{B, t}}{D_{A, t}+D_{B, t}}=s_{t} \frac{P_{A, t}}{D_{A, t}}+\left(1-s_{t}\right) \frac{P_{B, t}}{D_{B, t}} .
$$

The upper panel of Figure 1 depicts the price-dividend ratios in the calm and contagion state. The dependence on the dividend share is similar to the results of Cochrane, Longstaff, and Santa-Clara (2008). Small assets have very high price-dividend ratios since the agent would like to hold a diversified portfolio of shares of both trees. This leads to a high demand for shares of the small tree and thus to a high share price. The price-dividend ratio of the market is constant for a log-investor and U-shaped for $\gamma>1$.

Comparing the calm and contagion state shows that price-dividend ratios are the largest in the contagion state. In our example, the relative difference is about $4 \%$. At first glance, a higher asset price in the contagion state seems to be counterintuitive. It can however be explained by the value of the intertemporal elasticity of substitution that is below one. The investor reacts to bad investment opportunities by consuming a smaller fraction of his wealth. This in turn implies that the wealth-consumption ratio (i.e. in our case the price-dividend ratio of the market) is largest in the worse contagion state. The same argument applies to assets $\mathrm{A}$ and $\mathrm{B}{ }^{4}$

\footnotetext{
${ }^{4}$ For a relative risk aversion below one, we see the opposite effect. The results of a robustness check with $\gamma=2 / 3$ are given in Section 5.2.
} 


\subsection{Risk Exposures}

The asset price $P_{i, t}$ depends on the current dividends of both trees and on the state variable $p_{t}$. For its dynamic properties (such as the equity risk premium, local volatility, or correlations with other assets), the exposures with respect to the risk factors are crucial. The dynamics of $P_{i}(i=A, B, m)$ can be written as

$$
\begin{aligned}
\frac{d P_{i, t}}{P_{i, t-}}= & \frac{E\left[d P_{i, t}\right]}{P_{i, t-}}+\sigma_{i, A} d W_{t}^{A}+\sigma_{i, B} d W_{t}^{B} \\
& +\sum_{j=1,2,3} L_{i, A, j} d N_{t}^{A, j}+\sum_{j=1,2,3} L_{i, B, j} d N_{t}^{B, j}+L_{i, 4} d N_{t}^{4}
\end{aligned}
$$

where $\sigma_{i, j}$ is the sensitivity with respect to the Wiener processes $j \in\{A, B\}$. The variable $L_{i, k, j}$ denotes the sensitivity with respect to jumps of type $j$ in dividend $k(k=A, B$, $j=1,2,3)$, and $L_{i, 4}$ denotes the sensitivity with respect to jumps from the contagion state to the calm state.

Proposition 4 For an asset $i$ with dividend stream $D_{A}^{\alpha_{1}} D_{B}^{\alpha_{2}}$, the sensitivities with respect to the Wiener processes are

$$
\begin{aligned}
\sigma_{i, A}(s, p) & =\frac{\int_{-\infty}^{\infty} I(s, v)\left(\gamma s+\theta_{1}\left(v, \alpha_{1}, \alpha_{2}\right)\right) J\left(v, p, \alpha_{1}, \alpha_{2}\right) d v}{\int_{-\infty}^{\infty} I(s, v) J\left(v, p, \alpha_{1}, \alpha_{2}\right) d v} \sigma_{A} \\
\sigma_{i, B}(s, p) & =\frac{\int_{-\infty}^{\infty} I(s, v)\left(\gamma(1-s)+\theta_{2}\left(v, \alpha_{1}, \alpha_{2}\right)\right) J\left(v, p, \alpha_{1}, \alpha_{2}\right) d v}{\int_{-\infty}^{\infty} I(s, v) J\left(v, p, \alpha_{1}, \alpha_{2}\right) d v} \sigma_{B} .
\end{aligned}
$$

The sensitivities with respect to the Poisson processes $N^{k, j}(k=A, B, j=1,2,3)$ are

$$
L_{i, k, j}=\left(1+L_{A}\right)^{\alpha_{1} \tilde{\alpha}_{1}}\left(1+L_{B}\right)^{\alpha_{2} \tilde{\alpha}_{2}} \frac{\int_{-\infty}^{\infty} I\left(s_{t}, v\right) J\left(v, p_{t}, \alpha_{1}, \alpha_{2}\right) d v}{\int_{-\infty}^{\infty} I\left(s_{t-}, v\right) J\left(v, p_{t-}, \alpha_{1}, \alpha_{2}\right) d v}-1
$$

where $\tilde{\alpha}_{1}$ and $\tilde{\alpha}_{2}$ are defined such that $D_{A}^{\tilde{\alpha}_{1}} D_{B}^{\tilde{\alpha}_{2}}$ is the dividend process of asset $k$ (i.e. the asset which exhibits the respective dividend jump). The sensitivities with respect to the Poisson process $N^{4}$ are

$$
L_{i, 4}(s, p)=\frac{\int_{-\infty}^{\infty} I(s, v) J\left(v, 1, \alpha_{1}, \alpha_{2}\right) d v}{\int_{-\infty}^{\infty} I(s, v) J\left(v, 0, \alpha_{1}, \alpha_{2}\right) d v}-1
$$

Detailed formulas for each jump risk factor can be found in Appendix C.

The proof is given in Appendix C. 
The sensitivities of the market are weighted averages of the exposures of the single assets:

$$
\frac{d P_{m, t}}{P_{m, t-}}=s_{t-} \frac{P_{A, t-} / D_{A, t-}}{P_{m, t-} / D_{m, t-}} \frac{d P_{A, t}}{P_{A, t-}}+\left(1-s_{t-}\right) \frac{P_{B, t-} / D_{B, t-}}{P_{m, t-} / D_{m, t-}} \frac{d P_{B, t}}{P_{B, t-}} .
$$

The exposure of an asset to a risk factor depends on the respective exposure of its dividend and of its price-dividend ratio. For a constant price-dividend ratio, the price sensitivities are identical to those of the dividend (cash-flow effect). A stochastic price-dividend ratio drives a wedge between price and dividend sensitivities. From an economic point of view, these differences can be attributed to the dependence of the stochastic discount factor on the joint dynamics of both dividends and thus on the state variables $s_{t}$ and $p_{t}$ (sdf effect). The exposures for our benchmark parametrization are shown in Figure 2. The upper panel gives the diffusion exposures. The cash-flow channel generates an exposure equal to the diffusion volatility for the own dividend and zero for shocks in the other dividend (depicted by the black dotted lines in the figures). The sdf effect introduces some additional exposure. For a small or medium-sized tree, the price-dividend ratio is decreasing in its dividend share. A positive shock to the own dividend increases its dividend share and thus decreases its price-dividend ratio, which in turn dampens the exposure of the asset price to this shock. Vice versa, the same effect leads to a positive cross-exposure to innovations in the dividend of the other tree. Similarly, a large asset has an excess exposure to innovations in its own dividend, and a negative cross-exposure to innovations in the other tree. The reaction to 'normal' dividend jumps can be explained in exactly the same way. Finally, the reaction to jumps that change the state is given by the sum of the dividend effect just described and a state variable effect shown in the lower left panel of Figure 2. Since the price-dividend ratios are the largest in the contagion state, a jump into the contagion state, ceteris paribus, drives all prices up (by around $4 \%$ in our benchmark case). This increase more than offsets the dividend effect so that both assets have a positive total exposure to contagion-triggering jumps (shown in the third row in Figure 2). In turn, the prices decrease by around $4 \%$ if the economy reverts to the calm state. There is no dividend effect upon this event so that the respective exposures of both assets are approximately $-4 \%$. 


\subsection{Equity Premia and Expected Returns}

The expected return on asset $i$ consists of the expected price change and the dividend yield:

$$
E_{t}\left[d R_{i, t}\right]=\frac{E\left[d P_{i, t}\right]}{P_{i, t-}}+\frac{D_{i, t}}{P_{i, t-}} d t
$$

The expected excess return follows directly from the risk exposures analyzed in Section 3.4 and the market prices of risk given in Section 3.2.

Proposition 5 The expected excess return on an asset with dividend stream $D_{A}^{\alpha_{1}} D_{B}^{\alpha_{2}}$ is

$$
\begin{aligned}
\frac{1}{d t} E_{t}\left[d R_{i, t}\right]-r_{f, t}= & \sigma_{i, A} \gamma s_{t} \sigma_{A}+\sigma_{i, B} \gamma\left(1-s_{t}\right) \sigma_{B} \\
& +\sum_{j=1,2,3} L_{i, A, j}\left[1-\left(1+L_{A} s_{t}\right)^{-\gamma}\right] \lambda^{A, j} \\
& +\sum_{j=1,2,3} L_{i, B, j}\left[1-\left(1+L_{B}\left(1-s_{t}\right)\right)^{-\gamma}\right] \lambda^{B, j}
\end{aligned}
$$

The expected (excess) return on the market portfolio is a weighted average of the individual expected (excess) returns again:

$$
E_{t}\left[d R_{m, t}\right]=s_{t} \frac{P_{A, t-} / D_{A, t-}}{P_{m, t-} / D_{m, t-}} E_{t}\left[d R_{A, t}\right]+\left(1-s_{t}\right) \frac{P_{B, t-} / D_{B, t-}}{P_{m, t-} / D_{m, t-}} E_{t}\left[d R_{B, t}\right] .
$$

The first two terms in Proposition 5 capture the risk premium for dividend diffusion risk. Since an asset is mainly exposed to shocks in its own dividend, this premium increases in the market price of risk for these shocks and thus in the dividend share of the asset. The remaining terms capture the jump risk premia. Note that jumps back from the contagion state to the calm state are not priced and thus do not have an impact here. For the other jumps, we have shown in Section 3.2 that the investor earns a positive risk premium on a negative exposure to these jumps. He thus earns a positive premium on jumps that do not change the state (since the exposures are negative), while he has to pay some premium on jumps from the calm to contagion state (since the corresponding exposures are positive). For our benchmark case, the expected excess returns are given in the middle panel of Figure 1. In line with intuition, the expected excess return on an asset is monotonically increasing in its dividend share. The contribution of the asset to aggregate consumption risk is negligible if its dividend share tends to zero, and so the market prices of its risk factors tend to zero. Since an asset is mainly exposed to its own dividend risk factors, the total risk premium of small assets tends to zero as well. 
Expected excess returns increase upon entering the contagion state due to an increase in the jump intensities and thus in the amount of jump risk. Decomposing the risk premium into the premium for diffusion and jump risk, however, shows that the bulk of the expected excess return is due to diffusion risk. Since only a small part of the expected excess return stems from jump risk exposure, the risk premium increases only moderately in case of contagion.

\subsection{Local Volatilities}

The local variance of the asset price $P_{i}(i=A, B, m)$ follows from the exposures given in Section 3.4:

$$
\frac{1}{d t} \operatorname{Var}\left[\frac{d P_{i, t}}{P_{i, t-}}\right]=\sigma_{i, A}^{2}+\sigma_{i, B}^{2}+2 \rho \sigma_{i, A} \sigma_{i, B}+\sum_{j=1,2,3} L_{i, A, j}^{2} \lambda^{A, j}+\sum_{j=1,2,3} L_{i, B, j}^{2} \lambda^{B, j} .
$$

The upper panel in Figure 3 depicts the local volatilities in the calm (left graph) and in the contagion state (right graph). The volatilities are significantly greater in the contagion state than in the calm state because of the larger jump intensities in combination with the larger jump exposures.

For the individual assets, the degree of excess volatility depends on the dividend share and on the state. In the calm state, the volatility of a large asset exceeds the volatility of the corresponding dividend, while the opposite holds true for a small asset. In the contagion state, the volatilities are always larger than the dividend volatilities, irrespective of the size of the tree in our example.

The excess volatility of a large asset can be attributed to its additional exposure to shocks in its own dividend (see Section 3.4). For a small asset, the dampened exposure to shocks in its own dividend reduces its volatility as compared to the volatility of the dividends. Finally, jumps that change the state can also add to the local volatility. Both assets have positive excess exposures to jumps from the calm state to the contagion state. The resulting additional local volatility, however, is not large enough to result in excess volatility for all trees in the calm state. In the contagion state, the intensity for jumps back into the calm state is greater, which indeed induces excess volatility for all assets in our example.

The volatility of the market, i.e. the claim to aggregate consumption, is smallest if both trees have the same size since the volatility of aggregate consumption is lowest then. Furthermore, the volatility of the claim to aggregate consumption always exceeds the 
volatility of consumption since the properties of the market are similar to the properties of the large asset that exhibits excess volatility in both states.

\subsection{Local Correlations}

The local correlation between the prices of asset A and B is given by

$$
\operatorname{Corr}_{A, B, t}=\frac{\operatorname{Cov}_{t}\left(\frac{d P_{A, t}}{P_{A, t-}}, \frac{d P_{B, t}}{P_{B, t-}}\right)}{\sqrt{\operatorname{Var}_{t}\left[\frac{d P_{A, t}}{P_{A, t-}}\right] \operatorname{Var}_{t}\left[\frac{d P_{B, t}}{P_{B, t-}}\right]}}
$$

where the local variances have been derived in the previous subsection. The local covariance is obtained from the exposures:

$$
\begin{aligned}
\operatorname{Cov}_{t}\left(\frac{d P_{A, t}}{P_{A, t-}}, \frac{d P_{B, t}}{P_{B, t-}}\right)= & \sigma_{A, A} \sigma_{B, A} d t+\sigma_{A, B} \sigma_{B, B} d t+\rho \sigma_{A, A} \sigma_{B, B} d t+\rho \sigma_{A, B} \sigma_{B, A} d t \\
& +\sum_{j=1,2,3} L_{A, A, j} L_{B, A, j} \lambda^{A, j} d t+\sum_{j=1,2,3} L_{A, B, j} L_{B, B, j} \lambda^{B, j} d t \\
& +L_{A, 4} L_{B, 4} \lambda^{4} d t .
\end{aligned}
$$

The correlations between the market portfolio and an individual asset are determined analogously.

The results in the benchmark case are given in the middle row of Figure 3. The correlation between the market portfolio and an individual asset increases in the dividend share of that asset and becomes one if the asset dominates the economy. If the dividend share tends to zero, the correlation converges to the correlation between the two single assets.

The correlation between the individual assets is positive, even though the dividends are uncorrelated. This effect has already been documented by Cochrane, Longstaff, and SantaClara (2008). It is driven by the dependence of the stochastic discount factor on both dividends, which in turn induces an exposure of asset prices to risk factors that do not influence their own dividends. In our model, however, correlations are much larger in the limiting cases than in the setup of Cochrane, Longstaff, and Santa-Clara (2008). These larger correlations are caused by jumps triggering a regime switch, i.e. by the dependence of the prices on the state variable $p_{t}$. The simultaneous increase in the prices of both assets as soon as the economy enters the contagion state leads to a positive correlation of the asset prices in the calm state even if the dividend share of one asset tends to zero. Similarly, the possibility of a regime change back to the calm state gives rise to a positive local correlation between the asset prices in the contagion state. 
The larger jump exposures and larger jump intensities in the contagion state imply that the correlation increases if contagion hits the economy, which is often used as a definition for a contagion state. ${ }^{5}$ However, note that there is a subtle difference between realized (co-)variances and local (co-)variances. While the former increase only if a jump indeed happens (and are thus naturally larger if the economy is in the contagion state), the latter depend on the mere probability of a jump that is larger in the contagion state than in the calm state.

Finally, the lower panel of Figure 3 depicts the betas of the assets. If one of the assets dominates the economy, its beta converges to one since the beta of the market is one as well. If the dividend share of an asset goes to zero, then its beta decreases because it is carrying less systematic risk. Since the market beta is a weighted average of the betas of large and small trees, the beta of a large tree must be above one.

In line with the economic intuition behind contagion risk, the beta of a small stock does not go to zero in the limit which parallels the limiting behavior of the correlation. Economically, the small asset is still exposed to the risk of a regime change. Since the market portfolio has a significant exposure to this risk as well, the risk of a regime change is systematic, and the beta of the small asset cannot drop below a certain positive number. This lower boundary is larger in the contagion state than in the calm state which is well in line with the empirical evidence that, during economic crises, it becomes much harder to find a 'zero-beta' asset.

\section{Cross-sectional Pricing Effects of Contagion}

We now discuss the impact which the threat of contagion has on the cross-section of asset prices by relaxing the assumption of identical trees. In Section 4.1, we analyze the case where contagion mainly affects one asset while the jump intensity of the other asset increases only slightly. In Section 4.2, we compare an asset that never induces contagion with an asset that induces contagion whenever it is hit by a downward jump.

\footnotetext{
${ }^{5}$ For a detailed discussion of the different notions of contagion, interdependence and comovement, we refer the reader to the extensive analysis of Claessens and Forbes (2001).
} 


\subsection{Robust versus Excitable Assets}

We study an economy where, as in the benchmark case, all parameters are identical in the calm state. However, the two assets now differ in the contagion state. The column labeled 'Extension 1' of Table 1 summarizes the parameters. The jump intensity of asset $\mathrm{A}$ is doubled (0.8 instead of 0.4 in the calm state), whereas the jump intensity of asset B is even multiplied by 8 and is now 3.2. The overall intensity for a jump in the contagion state is thus still 4. Intuitively, we can think of asset A as a 'robust' asset, while asset B suffers much more from contagion and is called 'excitable' in the following.

The results for this calibration are shown in Figures 4, 5, 6, and 7. There are pronounced differences to the benchmark case in both states. Firstly, the price-dividend ratios shown in the upper row of Figure 4 are asymmetric in both states. The price of a small robust asset A is much larger than the price of an equally small excitable asset B. Stated differently, the excess demand for the small safe haven $\mathrm{A}$ is much larger than the excess demand for the very risky small asset B.

To understand the pricing implications of differences in the size as well as in the parameters of the trees, we separate the cash flow effects from the sdf effects. In a standard onetree setup, it is well known that the cash flow effect and the sdf effect go into opposite directions: A higher expected growth rate, e.g., increases future payoffs (leading to a larger price) and decreases the sdf (leading to a smaller price). For $\gamma>1$, the sdf effect usually dominates. In a two-tree setup with one dominating tree, the stochastic discount factor is mainly driven by this large asset (see Section 3.2). Therefore, the large asset is priced according to the intuition from the one-asset case, and the sdf effect dominates. This implies that the price of a large robust asset is lower than in the benchmark case since there is less risk in the economy. On the contrary, the price of a large excitable asset is greater because the economy is riskier.

The prices of small assets are driven by the sdf effect, which depends on the characteristics of the dominating asset, and the cash-flow effect, which depends on the characteristics of the small asset. The latter is no longer offset by the sdf effect, but can even prevail. As compared to the benchmark case, the price of a small excitable asset decreases due to the sdf effect (where the sdf is set by the large robust asset), and it also decreases due to the cash-flow effect (since the small asset is riskier than in the benchmark case). For a small robust asset, the intuition works just the other way round. Therefore, the price of a small excitable asset is smaller than in the benchmark case, while the price of a small robust asset is larger. 
The exposures of the asset prices to the risk factors are shown in Figure 5. The exposures to diffusion risk and to normal dividend jumps (i.e. jumps within a state) are rather similar to the benchmark case. The exposure to contagious jumps (i.e. jumps that change the state), however, is significantly different, which is mainly driven by the effect of the regime change on the price-dividend ratio (see lower right graphs of Figure 5). This effect and the impact of the dividend jump itself (shown in the second panel of Figure 5) add up to the total exposure to contagion-inducing jumps (shown in the lower left graphs of Figure 5).

The effect of the regime change can again be explained by separating the cash flow effect and the sdf effect. If the excitable asset $\mathrm{B}$ dominates the economy, consumption prospects become worse in the contagion state and the (positive) sdf effect of a change from the calm to the contagion state is larger than in the benchmark case. The opposite holds true if the robust asset A dominates. Therefore, the impact of a regime change on the prices of both assets is now asymmetric and depends on the dividend share $s_{t}$. It is much larger if the excitable asset $\mathrm{B}$ dominates than if the robust asset $\mathrm{A}$ dominates.

The cash-flow effect is driven by the fact that the expected dividend growth rate of the excitable asset drops more than that of the robust asset A. The effect is thus greater for an excitable asset than for a robust asset, and for small assets, it is not offset by the sdf effect. Consequently, the price-dividend ratio of a small excitable asset increases by less than the price-dividend ratio of the market, while the price-dividend ratio of a small robust asset increases by more than that of the market. In our numerical example, the difference is $2 \%$. Figure 6 depicts the excess exposures to the regime change. Furthermore, the negative cash flow effect for the excitable small asset B can even more than offset the positive sdf effect upon entering the contagion state. Indeed, the price of asset B drops if the economy enters the contagion state.

Assume that contagion hits an economy with a large excitable asset and a small robust asset that is a safe haven barely affected by contagion. The safe haven becomes much more valuable both in absolute terms and relatively to the market. There is only a small risk that its dividend level suddenly drops in the future. Drawing the analogy to a production economy, this can be interpreted as a stable production. This yields the classical flightto-quality pattern. If the economy eventually reverts to the calm state, this effect will, of course, trigger a price decrease of the safe haven relative to the market.

On the other hand, consider an economy with a small excitable asset (e.g. a small subsupplier) and a large robust asset (e.g. a global enterprise that is 'too big to fail'). Dete- 
riorating economic conditions are bad news for all assets. Policy-makers, however, might judge the impact of contagion on the large asset as very dangerous since this asset is responsible for a large part of the consumption in the economy. A common argument then goes that, in order to stabilize the economy, actions have to be taken to avoid additional losses in the large asset. On the other hand, small firms are usually considered to be of second-order importance. Therefore, their growth prospects suffer as soon as a crisis hits and their earnings are subject to several downward shocks, e.g. due to a freezing capital market or a slump in sales. The results of our model are in line with this anecdotal evidence: If the economy slips into the contagion state, the cash-flow effect leads to a significant decrease in the price of the small excitable asset both in absolute terms and relative to the market portfolio.

The risk-free rate is again smaller in the contagion state than in the calm state. The drop of interest rates is now the largest if the excitable asset B dominates the economy, while it is rather small if the robust asset A dominates. Following our line of argument above, this can be explained by the fact that the differences between the calm and the contagion state are much larger in the first case than in the second one.

The changes of the expected excess returns are much smaller than in the benchmark case. The equity premia in the contagion state decrease slightly as compared to the benchmark case if the robust asset $\mathrm{A}$ dominates the economy and increase if the excitable asset $\mathrm{B}$ dominates. To get the intuition, remember that the risk premia are mainly driven by diffusion risk. While the market prices of risk do not change, the exposures are more pronounced if the excitable asset $\mathrm{B}$ dominates, which in turn leads to larger equity risk premia. Furthermore, the contribution of jump risk to the equity risk premium, which is still quite small due to the moderate jump size, is greater if the excitable asset B dominates than if the robust asset $\mathrm{A}$ dominates.

A similar pattern can be observed for volatility. Although the overall picture is the same as in the benchmark calibration, the dependence of volatility on the dividend share and excess volatility are again more pronounced and the consequences of contagion are greater if the excitable asset $\mathrm{B}$ dominates than if the robust asset $\mathrm{A}$ dominates. Put differently, an increase in contagion risk - either because the intensity of jumps is larger in the contagion state or because a larger part of the economy is hit by contagion - leads to increased volatilities before contagion actually happens and, of course, to much higher volatilities once the economy is hit by contagion.

The correlations are again greater than in the benchmark case if the excitable asset B 
dominates and smaller if the safe haven A dominates. The reason is that correlations between very small and very large assets are mainly driven by their joint exposures to regime changes. Since these exposures are larger in absolute terms when asset B dominates, the correlation is larger in this case as well.

Finally, these results feed into the beta values of the assets: The small robust asset A carries a lot of systematic risk although it is not directly hit by contagion, which again follows from the sdf effect described above: If an excitable asset B dominates the economy (and the sdf), then its negative growth prospects in the contagion state 'spill over' to asset $\mathrm{A}$ via the stochastic discount factor. The resulting positive beta of asset $\mathrm{A}$ is documented in the empirical literature dealing with contagion and systemic risk. ${ }^{6}$ This fact is independent of whether asset $\mathrm{A}$ is small and itself relatively robust to contagion.

\subsection{Propensity to Trigger Contagion}

We now study the case where only one asset is able to trigger contagion. Column 'Extension 2' of Table 1 summarizes the corresponding parameters. In the calm state, the overall intensities for jumps in asset $\mathrm{A}$ or $\mathrm{B}$ are still 0.4 , and on average, every second jump induces contagion. While both assets are equally likely to induce contagion in the benchmark case, we now assume that only jumps of asset A can trigger contagion.

Figure 8 depicts our results. They are similar to those of the benchmark calibration: differences between the assets with respect to their robustness against contagion have more significant pricing implications than differences in their propensity to induce contagion. Furthermore, differences to the benchmark calibration - if any - are only observed in the calm state, while the results for the contagion state hardly differ. Since we have not changed any parameters in the contagion state, but only the propensities of the two trees to induce contagion (and not even their probabilities to suffer a downward jump), this result is perfectly in line with intuition.

A slight difference to the benchmark calibration exists for the equity risk premia in the calm state. They are now greater if asset $\mathrm{A}$ is small and smaller if asset $\mathrm{A}$ is large. The reason is that the equity risk premium mainly depends on the exposures to (systematic) risk factors in the large asset. If A dominates the economy, then only contagious jumps are priced, and the corresponding premium is negative (see Section 3.5). On the other

\footnotetext{
${ }^{6}$ See, e.g., Claessens and Forbes (2001) for a general overview.
} 
hand, if $\mathrm{A}$ is very small and B dominates, then there is only a jump risk premium for normal jumps. Since this premium is positive, both equity risk premia increase.

Furthermore, the difference between the assets in their propensity to induce contagion drives a wedge between the volatilities of asset A and asset B in the calm state. Volatilities of the contagion-inducing asset A are smaller, while volatilities of asset B are greater than in the benchmark calibration. To get the intuition, consider the exposures to normal jumps. Each asset has - in absolute terms - a large exposure to this type of jumps in its own dividend and a small cross-exposure to this type of jumps in the other tree. For contagious jumps, the opposite holds true. Since asset B exhibits only normal jumps and asset $\mathrm{A}$ exhibits only contagious jumps, the average exposure thus decreases in absolute terms for asset A and increases for asset B, and so does the volatility. Finally, the volatility of the market portfolio follows from the volatilities of the individual assets. It is thus smaller if the contagion-inducing asset $\mathrm{A}$ dominates and larger if asset $\mathrm{B}$ dominates the economy.

\section{Robustness Checks}

\subsection{Constant Dividend Growth}

In the benchmark case, the calm and contagion state differ both in the expected dividend growth rate and in jump intensities. As a robustness check, we analyze the case where the expected growth rates are identical in both states. The parameters are given in the column 'Equal Growth Rates' in Table 1. While the drift (conditional on no jumps) is still 0.03 in the calm state, it is set to 0.067 in the contagion state as to offset the greater expected losses.

The results for this calibration are shown in Figures 9 and 10. Most results are qualitatively very similar to the benchmark calibration. Overall, the risk in the economy is decreasing since the average growth rate no longer depends on the state of the economy. Therefore, volatilities and correlations are on average lower.

Compared to the benchmark case, the difference between the calm and contagion state is reduced. While the jump intensity is by definition greater in the contagion state the expected growth rate does not drop any more. Thus, the price-dividend ratios hardly change between the two states and the exposures to contagious jumps are very similar to 
the exposures to normal jumps. Furthermore, the price reaction to a jump back to the calm state is negligible.

The change in the exposures to regime changes has an impact on the second moments. First, volatilities are smaller than in the benchmark case, and the difference between the volatilities in the contagion and calm state is smaller as well. Second, correlations between very small and very large assets, which are mainly driven by the exposure to regime changes, are significantly lower and now close to zero.

\subsection{Relative Risk Aversion}

Our results are robust against a change in the relative risk aversion $\gamma$. As long as $\gamma>1$, there are no qualitative deviations from the benchmark case. With a relative risk aversion above one, the intertemporal elasticity of substitution is below one. As discussed earlier, this implies that price-dividend ratios are larger in the contagion than in the calm state. We have also solved our model for $\gamma=2 / 3$. As expected, price-dividend ratios are lower in the contagion than in the calm state, i.e. prices drop when the economy enters the contagion state. All the other results (in particular, the exposures to the risk factors) are qualitatively similar to the benchmark case.

Additionally, we have carried out robustness checks with respect to all other parameters (diffusion volatilities, drift rates, jump intensities, and the loss size). Prices and risk exposures are increasing in the overall economic risk and decreasing in the expected dividend growth rate where the dividend growth effect prevails. This has also been shown in the numerical examples above. The implications of contagion risk and the cross-sectional pricing results are qualitatively stable.

\section{Conclusion}

We have solved for the equilibrium in a two tree model where dividends are subject to contagion risk. Jumps do not only trigger instantaneous, persistent losses in dividends, but can also increase the probability of subsequent jumps across the whole economy. In the benchmark case with identical trees, the price-dividend ratios and asset pricing moments depend on the dividend share, as has been shown in Cochrane, Longstaff, and Santa-Clara (2008) and Martin (2009). 
In our model, however, there is also a dependence on the state of the economy. In the benchmark case, both assets react in the same direction if the regime changes. These joint exposures lead to excess volatilities of large assets in the calm state and further increase the volatilities of all assets in the contagion state. Furthermore, correlations between very small and very large assets as well as betas of very small assets do not converge to zero, but can be significantly positive. The mere fact that contagion risk is present in the economy gives rise to additional systematic risk in both assets.

The analysis of two non-identical trees allows us to assess the impact of contagion risk on the differential pricing of assets in the cross-section. We find significant differences between assets that are severely affected by contagion and assets that are more robust to systemic risk. Economically, this can be explained by the fact that the stochastic discount factor is mainly driven by the characteristics of the large asset. If a rather robust asset dominates the market, then a small, contagion-sensitive asset exhibits a downward jump when contagion occurs (in absolute terms as well as relative to the market). If, on the other hand, an excitable asset dominates the economy, then the price-dividend ratio of a small safe haven increases both in absolute terms and relatively to the market when the economy reaches the contagion state. On the contrary, the propensity to induce contagion has a very small effect on prices and price dynamics. The threat of contagion and its consequences for the various assets are thus of first-order importance, whereas it hardly matters which asset actually might trigger contagion.

One avenue for future research is to solve for the equilibrium with Epstein-Zin utility. This would allow us to choose a relative risk aversion and an intertemporal elasticity of substitution both greater than one. Furthermore, a preference for early resolution of uncertainty would result in a risk premium for the state variable $p_{t}$ as well so that there would indeed be a nonzero 'market price of contagion risk'. Another interesting extension is option pricing. While it is obvious that downward jumps induce a volatility smile, the implications of a regime change for its level and slope might be more involved. 


\section{A Proof of Proposition 1 and 2}

The risk-free rate and the market prices of risk follow from the dynamics of the pricing kernel $\xi_{t}$ which is given by $\xi_{t}=e^{-\delta t} \frac{\left(D_{A, t}+D_{B, t}\right)^{-\gamma}}{\left(D_{A, 0}+D_{B, 0}\right)^{-\gamma}}$. Ito's Lemma yields

$$
\begin{aligned}
\frac{d \xi_{t}}{\xi_{t-}}=[ & -\delta-\gamma s_{t}\left(\mu_{A}+0.5 \sigma_{A}^{2}\right)-\gamma\left(1-s_{t}\right)\left(\mu_{B}+0.5 \sigma_{B}^{2}\right) \\
& \left.+0.5 \gamma(1+\gamma)\left(s_{t}^{2} \sigma_{A}^{2}+\left(1-s_{t}\right)^{2} \sigma_{B}^{2}+2 s_{t}\left(1-s_{t}\right) \rho \sigma_{A} \sigma_{B}\right)\right] d t \\
& -\gamma s_{t} \sigma_{A} d W_{t}^{A}-\gamma\left(1-s_{t}\right) \sigma_{B} d W_{t}^{B} \\
& +\sum_{j=1,2,3}\left[\left(1+L_{A} s_{t}\right)^{-\gamma}-1\right] d N_{t}^{A, j}+\sum_{j=1,2,3}\left[\left(1+L_{B}\left(1-s_{t}\right)\right)^{-\gamma}-1\right] d N_{t}^{B, j}
\end{aligned}
$$

where $s_{t}$ denotes the dividend share of asset $A$ at time $t$. The risk-free rate $r_{f, t}$ is given by

$$
r_{f, t} d t=-E_{t}\left[\frac{d \xi_{t}}{\xi_{t-}}\right]
$$

The market prices of risk follow from the sensitivities of the pricing kernel with respect to the different risk factors.

\section{B Proof of Proposition 3}

The full version of Proposition 3 reads as follows.

Proposition 6 The price-dividend ratio of an asset with dividend stream $D_{A}^{\alpha_{1}} D_{B}^{\alpha_{2}}$ is

$$
\frac{P_{t}}{D_{A, t}^{\alpha_{1}} D_{B, t}^{\alpha_{2}}}=\int_{-\infty}^{\infty} I\left(s_{t}, v\right) J\left(v, p_{t}\right) d v
$$

where the functions $I$ and $J$ are given by

$$
\begin{aligned}
& I(s, v)=\left(\frac{1}{\sqrt{s(1-s)}}\right)^{\gamma}\left(\frac{1-s}{s}\right)^{i v}=\left(D_{A}+D_{B}\right)^{\gamma} D_{A}^{-\frac{\gamma}{2}-i v} D_{B}^{-\frac{\gamma}{2}+i v} \\
& J(v, p)=\frac{2 F(v)}{1+M_{2}(\theta, p)}\left(\frac{1}{M_{1}(\theta)+Z(\theta)-2 \delta}+\frac{M_{2}(\theta, p)}{M_{1}(\theta)-Z(\theta)-2 \delta}\right)
\end{aligned}
$$

The function $F$ is given by

$$
F(v)=\frac{1}{2 \pi} \frac{\Gamma\left(\frac{\gamma}{2}+i v\right) \Gamma\left(\frac{\gamma}{2}-i v\right)}{\Gamma(\gamma)}
$$


and the argument $\theta$ is given by $\theta=\left(\theta_{1}, \theta_{2}, \theta_{3}\right)=\left(\alpha_{1}-\frac{\gamma}{2}-i v, \alpha_{2}-\frac{\gamma}{2}+i v, 0\right), v \in \mathbb{R}$. The functions $M_{1}, M_{2}$, and $Z$ are defined as follows:

$$
\begin{aligned}
M_{1}(\theta)= & \theta_{1}\left(\mu_{A}^{\text {calm }}+\mu_{A}^{\text {cont }}\right)+\theta_{2}\left(\mu_{B}^{\text {calm }}+\mu_{B}^{\text {cont }}\right)+\theta_{1}^{2} \sigma_{A}^{2}+\theta_{2}^{2} \sigma_{B}^{2}+2 \theta_{1} \theta_{2} \rho \sigma_{A} \sigma_{B} \\
& +\lambda_{A}^{\text {calm,calm }}\left(\left(1+L_{A}\right)^{\theta_{1}}-1\right)+\lambda_{B}^{\text {calm,calm }}\left(\left(1+L_{B}\right)^{\theta_{2}}-1\right) \\
& +\lambda_{A}^{\text {cont,cont }}\left(\left(1+L_{A}\right)^{\theta_{1}}-1\right)+\lambda_{B}^{\text {cont }, \text { cont }}\left(\left(1+L_{B}\right)^{\theta_{2}}-1\right) \\
& -\lambda_{A}^{\text {calm,cont }}-\lambda_{B}^{\text {calm }, \text { cont }}-\lambda^{\text {cont }, \text { calm }} \\
M_{2}(\theta, p)= & \frac{2 Z(\theta)}{2 e^{\theta_{3} K_{3}+K_{1}(\theta)+Z(\theta)}} \cdot p+\frac{2 Z(\theta)}{-2 e^{-\theta_{3}} K_{2}(\theta)-K_{1}(\theta)+Z(\theta)} \cdot(1-p)-1 \\
Z(\theta)= & \sqrt{K_{1}(\theta)^{2}-4 K_{2}(\theta) K_{3}}
\end{aligned}
$$

where the functions $K_{1}, K_{2}$ and $K_{3}$ are given by

$$
\begin{aligned}
K_{1}(\theta)= & \left(\mu_{A}^{\text {cont }}-\mu_{A}^{\text {calm }}\right) \theta_{1}+\left(\mu_{B}^{\text {cont }}-\mu_{B}^{\text {calm }}\right) \theta_{2} \\
& -\lambda_{A}^{\text {calm }, \text { calm }}\left(\left(1+L_{A}\right)^{\theta_{1}}-1\right)-\lambda_{B}^{\text {calm,calm }}\left(\left(1+L_{B}\right)^{\theta_{2}}-1\right) \\
& +\lambda_{A}^{\text {cont }, \text { cont }}\left(\left(1+L_{A}\right)^{\theta_{1}}-1\right)+\lambda_{B}^{\text {cont }, \text { cont }}\left(\left(1+L_{B}\right)^{\theta_{2}}-1\right) \\
& -\lambda^{\text {cont }, \text { calm }}+\lambda_{A}^{\text {calm,cont }}+\lambda_{B}^{\text {calm,cont }} \\
K_{2}(\theta)= & -\lambda_{A}^{\text {calm,cont }}\left(1+L_{A}\right)^{\theta_{1}}-\lambda_{B}^{\text {calm,cont }}\left(1+L_{B}\right)^{\theta_{2}} \\
K_{3}= & \lambda^{\text {cont }, \text { calm }} .
\end{aligned}
$$

Proof: W.l.o.g., the current time is denoted by 0 . The following expectation can be obtained directly from Duffie, Pan, and Singleton (2000) (p. 1351 and p. 1371) since the model is affine:

$$
E_{0}\left[\exp \left(\left(\theta_{1}, \theta_{2}, \theta_{3}\right) \cdot\left(\begin{array}{c}
y_{A, T} \\
y_{B, T} \\
p_{T}
\end{array}\right)\right)\right]=\exp \left(\alpha_{T, \theta_{1}, \theta_{2}, \theta_{3}}(0)+\beta_{T, \theta_{1}, \theta_{2}, \theta_{3}}(0)\left(\begin{array}{c}
y_{A, 0} \\
y_{B, 0} \\
p_{0}
\end{array}\right)\right) .
$$

Duffie, Pan, and Singleton (2000) show that $\alpha_{T, \theta_{1}, \theta_{2}, \theta_{3}}$ and $\beta_{T, \theta_{1}, \theta_{2}, \theta_{3}}$ satisfy the following ordinary differential equations with boundary conditions $\beta(T)=\left(\theta_{1}, \theta_{2}, \theta_{3}\right)^{\prime}$ and $\alpha(T)=0$. For the 
sake of simplicity, the subscripts $\left(T, \theta_{1}, \theta_{2}, \theta_{3}\right)$ are omitted from now on.

$$
\begin{aligned}
& \dot{\beta}_{1}(t)=0 \\
& \dot{\beta}_{2}(t)=0 \\
& \dot{\beta}_{3}(t)=\left(\mu_{A}^{c o n t}-\mu_{A}^{c a l m}\right) \beta_{1}(t)+\left(\mu_{B}^{c o n t}-\mu_{B}^{c a l m}\right) \beta_{2}(t) \\
& -\lambda_{A}^{\text {calm,calm }}\left(e^{\beta_{1}(t) \log \left(1+L_{A}\right)}-1\right)-\lambda_{B}^{\text {calm,calm }}\left(e^{\beta_{2}(t) \log \left(1+L_{B}\right)}-1\right) \\
& -\lambda_{A}^{\text {calm,cont }}\left(e^{\beta_{1}(t) \log \left(1+L_{A}\right)-\beta_{3}(t)}-1\right)-\lambda_{B}^{\text {calm,cont }}\left(e^{\beta_{2}(t) \log \left(1+L_{B}\right)-\beta_{3}(t)}-1\right) \\
& +\lambda_{A}^{\text {cont,cont }}\left(e^{\beta_{1}(t) \log \left(1+L_{A}\right)}-1\right)+\lambda_{B}^{\text {cont,cont }}\left(e^{\beta_{2}(t) \log \left(1+L_{B}\right)}-1\right)+\lambda^{\text {cont,calm }}\left(e^{\beta_{3}(t)}-1\right) \\
& \dot{\alpha}(t)=-\mu_{A}^{\text {cont }} \beta_{1}(t)-\mu_{B}^{c o n t} \beta_{2}(t)-\frac{1}{2} \beta_{1}(t)^{2} \sigma_{A}^{2}-\frac{1}{2} \beta_{2}(t)^{2} \sigma_{B}^{2}-\beta_{1}(t) \beta_{2}(t) \rho \sigma_{A} \sigma_{B} \\
& -\lambda_{A}^{\text {cont,cont }}\left(e^{\beta_{1}(t) \log \left(1+L_{A}\right)}-1\right)-\lambda_{B}^{\text {cont,cont }}\left(e^{\beta_{2}(t) \log \left(1+L_{B}\right)}-1\right)-\lambda^{\text {cont }, \text { calm }}\left(e^{\beta_{3}(t)}-1\right)
\end{aligned}
$$

The boundary condition for $\beta(T)$ yields $\beta_{1}(t)=\theta_{1}$ and $\beta_{2}(t)=\theta_{2}$. Thus, the system of four equations simplifies to a system of two equations:

$$
\begin{aligned}
\dot{\beta}_{3}(t)= & \left(\mu_{A}^{\text {cont }}-\mu_{A}^{\text {calm }}\right) \theta_{1}+\left(\mu_{B}^{\text {cont }}-\mu_{B}^{\text {calm }}\right) \theta_{2} \\
& -\lambda_{A}^{\text {calm,calm }}\left(e^{\theta_{1} \log \left(1+L_{A}\right)}-1\right)-\lambda_{B}^{\text {calm,calm }}\left(e^{\theta_{2} \log \left(1+L_{B}\right)}-1\right) \\
& -\lambda_{A}^{\text {calm,cont }}\left(e^{\theta_{1} \log \left(1+L_{A}\right)-\beta_{3}(t)}-1\right)-\lambda_{B}^{\text {calm,cont }}\left(e^{\theta_{2} \log \left(1+L_{B}\right)-\beta_{3}(t)}-1\right) \\
& +\lambda_{A}^{\text {cont }, \text { cont }}\left(e^{\theta_{1} \log \left(1+L_{A}\right)}-1\right)+\lambda_{B}^{\text {cont }, \text { cont }}\left(e^{\theta_{2} \log \left(1+L_{B}\right)}-1\right)+\lambda^{\text {cont }, \text { calm }}\left(e^{\beta_{3}(t)}-1\right) \\
\dot{\alpha}(t)= & -\mu_{A}^{\text {cont }} \theta_{1}-\mu_{B}^{\text {cont }} \theta_{2}-\frac{1}{2} \theta_{1}^{2} \sigma_{A}^{2}-\frac{1}{2} \theta_{2}^{2} \sigma_{B}^{2}-\theta_{1} \theta_{2} \rho \sigma_{A} \sigma_{B} \\
& -\lambda_{A}^{\text {cont }, \text { cont }}\left(e^{\theta_{1} \log \left(1+L_{A}\right)}-1\right)-\lambda_{B}^{\text {cont,cont }}\left(e^{\theta_{2} \log \left(1+L_{B}\right)}-1\right)-\lambda^{\text {cont,calm }}\left(e^{\beta_{3}(t)}-1\right)
\end{aligned}
$$

with boundary conditions $\beta_{3}(T)=\theta_{3}$ and $\alpha(T)=0$. Lemma 7 gives the solution for $\beta_{3}(t)$.

Lemma 7 The function $\beta_{3}(t)$ is given by

$$
\begin{aligned}
\beta_{3}(t)= & \theta_{3}+\log \left[\left(-2 e^{-\theta_{3}} K_{2}(\theta)-K_{1}(\theta)+Z(\theta)\right)\left(e^{-Z(\theta)(t-T)}-1\right)+2 Z(\theta)\right] \\
& -\log \left[\left(2 e^{\theta_{3}} K_{3}+K_{1}(\theta)+Z(\theta)\right)\left(e^{-Z(\theta)(t-T)}-1\right)+2 Z(\theta)\right]+2 \pi i n \\
\text { where } K_{1}(\theta)= & \left(\mu_{A}^{\text {cont }}-\mu_{A}^{\text {calm }}\right) \theta_{1}+\left(\mu_{B}^{\text {cont }}-\mu_{B}^{\text {calm }}\right) \theta_{2} \\
& -\lambda_{A}^{\text {calm,calm }}\left(e^{\theta_{1} \log \left(1+L_{A}\right)}-1\right)-\lambda_{B}^{\text {calm,calm }}\left(e^{\theta_{2} \log \left(1+L_{B}\right)}-1\right) \\
& +\lambda_{A}^{\text {cont }, \text { cont }}\left(e^{\theta_{1} \log \left(1+L_{A}\right)}-1\right)+\lambda_{B}^{\text {cont }, \text { cont }}\left(e^{\theta_{2} \log \left(1+L_{B}\right)}-1\right) \\
& -\lambda^{\text {cont }, \text { calm }}+\lambda_{A}^{\text {calm,cont }}+\lambda_{B}^{\text {calm,cont }} \\
K_{2}(\theta)= & -\lambda_{A}^{\text {calm,cont }} e^{\theta_{1} \log \left(1+L_{A}\right)}-\lambda_{B}^{\text {calm,cont }} e^{\theta_{2} \log \left(1+L_{B}\right)} \\
K_{3}= & \lambda^{\text {cont,calm }} \\
Z(\theta)= & \sqrt{K_{1}(\theta)^{2}-4 K_{2}(\theta) K_{3} .} .
\end{aligned}
$$


Proof: Let $K_{1}, K_{2}$ and $K_{3}$ be defined as in Lemma 7 . Then the ODE (2) reads

$$
\dot{\beta}_{3}(t)=K_{1}+K_{2} e^{-\beta_{3}(t)}+K_{3} e^{\beta_{3}(t)}
$$

Multiplying with $e^{\beta_{3}(t)}$ and defining $g(t)=e^{\beta_{3}(t)}$ gives the following equivalent Riccati boundary problem for $g(t)$ :

$$
\dot{g}(t)=K_{2}+K_{1} g(t)+K_{3} g(t)^{2}
$$

with boundary condition $g(T)=e^{\theta_{3}}$. A straightforward calculation shows that, for solving the Riccati problem, solving the following linear boundary problem is sufficient:

$$
\begin{aligned}
\ddot{h}(t)-K_{1} \dot{h}(t)+K_{2} K_{3} h(t) & =0 \\
\dot{h}(T) & =h_{1} \\
h(T) & =h_{0}
\end{aligned}
$$

where the boundary values fulfil the relation

$$
e^{\theta_{3}}=-\frac{h_{1}}{K_{3} h_{0}} .
$$

If there exists an open neighborhood $D$ of $T$ which does not contain any zeros of $h$, then $g(t)=-\frac{\dot{h}(t)}{K_{3} h(t)}$ is well-defined on $D$ and solves the Riccati problem (4) on the domain $D$.

Abbreviating $\sqrt{K_{1}^{2}-4 K_{2} K_{3}}=Z$, the zeroes of the characteristic polynomial of (5) are

$$
\frac{K_{1}}{2}-\sqrt{\frac{K_{1}^{2}}{4}-K_{2} K_{3}}=\frac{K_{1}}{2}-\frac{Z}{2} \quad \text { and } \quad \frac{K_{1}}{2}+\sqrt{\frac{K_{1}^{2}}{4}-K_{2} K_{3}}=\frac{K_{1}}{2}+\frac{Z}{2} .
$$

These are of order 1 and, therefore, the general solution of the second-order homogeneous boundary problem (5) equals

$$
h(t)=K_{4} e^{\left(\frac{K_{1}}{2}-\frac{Z}{2}\right)(t-T)}+K_{5} e^{\left(\frac{K_{1}}{2}+\frac{Z}{2}(t-T)\right)}
$$

where the constants $K_{4}$ and $K_{5}$ depend on the boundary values $h_{1}$ and $h_{0}$. Choosing $h_{0}=1$ and $h_{1}=-e^{\theta_{3}} K_{3}$ (so that the constraint (6) is met) results in

$$
\begin{aligned}
1 & =K_{4}+K_{5} \\
-e^{\theta_{3}} K_{3} & =K_{4}\left(\frac{K_{1}}{2}-\frac{Z}{2}\right)+K_{5}\left(\frac{K_{1}}{2}+\frac{Z}{2}\right)
\end{aligned}
$$

which can be solved for $K_{4}$ and $K_{5}$ :

$$
\begin{aligned}
& K_{4}=\frac{e^{\theta_{3}} K_{3}+\frac{K_{1}}{2}+\frac{Z}{2}}{Z}=\frac{2 e^{\theta_{3}} K_{3}+K_{1}+Z}{2 Z} \\
& K_{5}=1-K_{4}=\frac{Z-2 e^{\theta_{3}} K_{3}-K_{1}}{2 Z} .
\end{aligned}
$$


Note that the function $h$ has no zeros in a neighborhood of $T$ (since $h(T)=1$ and $h$ is continuous) so that the solution $g$ of our original Riccati problem (4) equals

$$
\begin{aligned}
g(t)=-\frac{\dot{h}(t)}{K_{3} h(t)} & =-\frac{\left(\frac{K_{1}}{2}-\frac{Z}{2}\right) K_{4} e^{\left(\frac{K_{1}}{2}-\frac{Z}{2}\right)(t-T)}+\left(\frac{K_{1}}{2}+\frac{Z}{2}\right)\left(1-K_{4}\right) e^{\left(\frac{K_{1}}{2}+\frac{Z}{2}\right)(t-T)}}{K_{3} K_{4} e^{\left(\frac{K_{1}}{2}-\frac{Z}{2}\right)(t-T)}+K_{3}\left(1-K_{4}\right) e^{\left(\frac{K_{1}}{2}+\frac{Z}{2}\right)(t-T)}} \\
& =-\frac{\left(K_{1}-Z\right) K_{4} e^{-Z(t-T)}+\left(K_{1}+Z\right)\left(1-K_{4}\right)}{2 K_{3} K_{4} e^{-Z(t-T)}+2 K_{3}\left(1-K_{4}\right)} \\
& =\frac{\left(-2 K_{2}-e^{\theta_{3}} K_{1}+e^{\theta_{3}} Z\right)\left(e^{-Z(t-T)}-1\right)+2 Z e^{\theta_{3}}}{\left(2 e^{\theta_{3}} K_{3}+K_{1}+Z\right)\left(e^{-Z(t-T)}-1\right)+2 Z} \\
& =e^{\theta_{3}} \frac{\left(-2 e^{-\theta_{3}} K_{2}-K_{1}+Z\right)\left(e^{-Z(t-T)}-1\right)+2 Z}{\left(2 e^{\theta_{3}} K_{3}+K_{1}+Z\right)\left(e^{-Z(t-T)}-1\right)+2 Z} .
\end{aligned}
$$

Note that, for $g(T)=0$, equation (8) provides the classical solution for Riccati equations given, e.g., in Liu and Pan (2003). In our case, however, $g(T)=e^{\theta_{3}} \neq 0$.

Taking logarithms on both sides, we get the final result for $\beta_{3}$ (note that the logarithm we take here need not be the principal value of the complex logarithm, but can be any branch of the complex logarithm; the extra $2 \pi i n$ will cancel out in the integration later on anyway). This completes the proof of Lemma 7.

The solution for $\alpha$ (given in Lemma 8) follows from Lemma 7 through integration.

Lemma 8 The function $\alpha$ is given by

$$
\begin{aligned}
\alpha(t)= & {\left[-\mu_{A}^{\text {cont }} \theta_{1}-\mu_{B}^{c o n t} \theta_{2}-\frac{1}{2} \theta_{1}^{2} \sigma_{A}^{2}-\frac{1}{2} \theta_{2}^{2} \sigma_{B}^{2}-\theta_{1} \theta_{2} \rho \sigma_{A} \sigma_{B}\right.} \\
& \left.-\lambda_{A}^{\text {cont }, \text { cont }}\left(e^{\theta_{1} \log \left(1+L_{A}\right)}-1\right)-\lambda_{B}^{\text {cont }, \text { cont }}\left(e^{\theta_{2} \log \left(1+L_{B}\right)}-1\right)+\lambda^{\text {cont }, \text { calm }}\right](t-T) \\
& +\log \left(\left(2 e^{\theta_{3}} K_{3}+K_{1}(\theta)+Z(\theta)\right)\left(e^{-Z(\theta)(t-T)}-1\right)+2 Z(\theta)\right) \\
& +\frac{K_{1}(\theta)+Z(\theta)}{2}(t-T)-\log (2 Z(\theta))+2 \pi i n
\end{aligned}
$$

where $K_{1}(\theta), K_{2}(\theta), K_{3}$ and $Z(\theta)$ have been defined in Lemma $\%$.

Proof: Plugging $g(t)=e^{\beta_{3}(t)}$ into the ODE (3) results in the following ODE for $\alpha$ (with boundary condition $\alpha(T)=0$ )

$$
\begin{aligned}
\dot{\alpha}(t)= & -\mu_{A}^{\text {cont }} \theta_{1}-\mu_{B}^{\text {cont }} \theta_{2}-\frac{1}{2} \theta_{1}^{2} \sigma_{A}^{2}-\frac{1}{2} \theta_{2}^{2} \sigma_{B}^{2}-\theta_{1} \theta_{2} \rho \sigma_{A} \sigma_{B} \\
& -\lambda_{A}^{\text {cont }, \text { cont }}\left(e^{\theta_{1} \log \left(1+L_{A}\right)}-1\right)-\lambda_{B}^{\text {cont,cont }}\left(e^{\theta_{2} \log \left(1+L_{B}\right)}-1\right)+\lambda^{\text {cont }, \text { calm }}-\lambda^{\text {cont }, \text { calm }} g(t) .
\end{aligned}
$$


Integration then yields (since $\alpha(T)=0)$

$$
\begin{aligned}
\alpha(t)=\int_{T}^{t} \dot{\alpha}(u) d u= & (t-T)\left[-\mu_{A}^{\text {cont }} \theta_{1}-\mu_{B}^{\text {cont }} \theta_{2}-\frac{1}{2} \theta_{1}^{2} \sigma_{A}^{2}-\frac{1}{2} \theta_{2}^{2} \sigma_{B}^{2}-\theta_{1} \theta_{2} \rho \sigma_{A} \sigma_{B}\right. \\
& \left.-\lambda_{A}^{\text {cont }, \text { cont }}\left(e^{\theta_{1} \log \left(1+L_{A}\right)}-1\right)-\lambda_{B}^{\text {cont }, \text { cont }}\left(e^{\theta_{2} \log \left(1+L_{B}\right)}-1\right)+\lambda^{\text {cont }, \text { calm }}\right] \\
& -\lambda^{\text {cont }, \text { calm }} \int_{T}^{t} g(u) d u .
\end{aligned}
$$

Plugging in the representation (7) for $g$ gives

$$
\begin{array}{r}
-K_{3} \int_{T}^{t} g(u) d u=\int_{T}^{t} \frac{\dot{h}(u)}{h(u)} d u=(\log h(t)-\log h(T))=\log \left(K_{4} e^{\left(\frac{K_{1}}{2}-\frac{Z}{2}\right)(t-T)}+K_{5} e^{\left(\frac{K_{1}}{2}+\frac{Z}{2}(t-T)\right)}\right) \\
=\frac{K_{1}+Z}{2}(t-T)+\log \left(\left(2 e^{\theta_{3}} K_{3}+K_{1}+Z\right)\left(e^{-Z(t-T)}-1\right)+2 Z\right)-\log (2 Z)+2 \pi i n
\end{array}
$$

where $n \in \mathbb{Z}$ stands for some unknown integer (not necessarily the same as in Lemma 7). This completes the proof of Lemma 8.

Neither $\beta$ nor $\alpha$ depend on $y_{A}, y_{B}$ or $p$. Both functions only depend on $t, T, \theta_{1}, \theta_{2}, \theta_{3}$ and the model parameters. Therefore,

$$
E_{0}\left[\exp \left(\left(\theta_{1}, \theta_{2}, \theta_{3}\right) \cdot\left(\begin{array}{c}
y_{A, T}-y_{A, 0} \\
y_{B, T}-y_{B, 0} \\
p_{T}-p_{0}
\end{array}\right)\right)\right]=e^{\chi\left(\theta_{1}, \theta_{2}, \theta_{3}, T, p_{0}\right)}
$$

where

$\chi\left(\theta_{1}, \theta_{2}, \theta_{3}, T, p_{0}\right)=\alpha_{T, \theta_{1}, \theta_{2}, \theta_{3}}(0)+\left[\beta_{T, \theta_{1}, \theta_{2}, \theta_{3}}(0)-\left(\begin{array}{c}\theta_{1} \\ \theta_{2} \\ \theta_{3}\end{array}\right)^{\prime}\right]\left(\begin{array}{c}y_{A, 0} \\ y_{B, 0} \\ p_{0}\end{array}\right)=\alpha(0)+\left(\beta_{3}(0)-\theta_{3}\right) p_{0}$.

The last equality holds because $\beta_{1}=\theta_{1}$ and $\beta_{2}=\theta_{2}$.

In some cases (especially if there is no latent state variable), $\chi\left(\theta_{1}, \theta_{2}, \theta_{3}, T, p_{0}\right)$ can be rewritten in the form $c\left(\theta_{1}, \theta_{2}, \theta_{3}\right) T$ where $c$ is called the cumulant-generating function. E.g., the approach of Martin (2009) is based on this cumulant-generating function. Our problem is a bit more involved since we have state variables. As the function $\chi$ is an affine function of $p_{0}$, however, the generalized solution of Branger, Schlag, and Wu (2011) applies here. According to Branger, Schlag, and $\mathrm{Wu}$ (2011), the current price-dividend ratio of an asset with dividend payments equal to $D_{A}^{\alpha_{1}} D_{B}^{\alpha_{2}}$ is given by

$$
\frac{P_{\alpha, 0}}{D_{\alpha, 0}}=\left(2 \cosh \frac{y_{B, 0}-y_{A, 0}}{2}\right)^{\gamma} \int_{-\infty}^{\infty} e^{i v\left(y_{B, 0}-y_{A, 0}\right)} F(v) \int_{0}^{\infty} e^{-\delta t+\chi\left(\alpha_{1}-\frac{\gamma}{2}-i v, \alpha_{2}-\frac{\gamma}{2}+i v, 0, t, p_{0}\right)} d t d v
$$


where $F$ is defined as

$$
F(v)=\frac{1}{2 \pi} \frac{\Gamma\left(\frac{\gamma}{2}+i v\right) \Gamma\left(\frac{\gamma}{2}-i v\right)}{\Gamma(\gamma)}
$$

Using that $y_{B, 0}-y_{A, 0}=\log \frac{1-s_{0}}{s_{0}}$, this can be rewritten as

$$
\frac{P_{\alpha, 0}}{D_{\alpha, 0}}=\left(\frac{1}{\sqrt{s_{0}\left(1-s_{0}\right)}}\right)^{\gamma} \int_{-\infty}^{\infty}\left(\frac{1-s_{0}}{s_{0}}\right)^{i v} F(v) \int_{0}^{\infty} e^{-\delta t+\chi\left(\alpha_{1}-\frac{\gamma}{2}-i v, \alpha_{2}-\frac{\gamma}{2}+i v, 0, t, p_{0}\right)} d t d v
$$

In the case with diffusion risk only and without state variables, a closed-form solution is available and proven by Martin (2009). Here, a closed-form solution seems impossible: in order to compute the outer integral via the Residue Theorem, one would need information about the poles of the integrand which lie in the upper complex halfplane. However, since the inner integral in (9) does not depend on $y_{A}$ or $y_{B}$ at all, this integral can be given in closed form.

\section{Lemma 9 It holds that}

$$
\int_{0}^{\infty} e^{-\delta t+\chi\left(\theta_{1}, \theta_{2}, \theta_{3}, t, p\right)} d t=-\frac{1}{\left(1+M_{2}(\theta, p)\right)\left(M_{1}(\theta)+Z(\theta)-2 \delta\right)}-\frac{M_{2}(\theta, p)}{\left(1+M_{2}(\theta, p)\right)\left(M_{1}(\theta)-Z(\theta)-2 \delta\right)}
$$

where $M_{1}$ and $M_{2}$ are defined as

$$
\begin{aligned}
M_{1}(\theta)= & \left(\mu_{A}^{\text {calm }}+\mu_{A}^{\text {cont }}\right) \theta_{1}+\left(\mu_{B}^{\text {calm }}+\mu_{B}^{\text {cont }}\right) \theta_{2}+\theta_{1}^{2} \sigma_{A}^{2}+\theta_{2}^{2} \sigma_{B}^{2}+2 \theta_{1} \theta_{2} \rho \sigma_{A} \sigma_{B} \\
& +\lambda_{A}^{\text {calm,calm }}\left(e^{\theta_{1} \log \left(1+L_{A}\right)}-1\right)+\lambda_{B}^{\text {calm,calm }}\left(e^{\theta_{2} \log \left(1+L_{B}\right)}-1\right) \\
& +\lambda_{A}^{\text {cont }, \text { cont }}\left(e^{\theta_{1} \log \left(1+L_{A}\right)}-1\right)+\lambda_{B}^{\text {cont,cont }}\left(e^{\theta_{2} \log \left(1+L_{B}\right)}-1\right) \\
& -\lambda_{A}^{\text {calm,cont }}-\lambda_{B}^{\text {calm,cont }}-\lambda^{\text {cont }, \text { calm }} \\
M_{2}(\theta, p)= & \frac{2 Z(\theta)}{-2 e^{-\theta_{3}} K_{2}(\theta)-K_{1}(\theta)+Z(\theta)} \cdot p+\frac{2 Z(\theta)}{2 e^{\theta_{3}} K_{3}+K_{1}(\theta)+Z(\theta)} \cdot(1-p)-1 .
\end{aligned}
$$

Proof: With Lemma 7 and 8, $\chi$ fulfils

$$
\begin{aligned}
& \chi\left(\theta_{1}, \theta_{2}, \theta_{3}, T, p\right)=\alpha(0)+\left(\beta_{3}(0)-\theta_{3}\right) p \\
= & {\left[\mu_{A}^{\text {cont }} \theta_{1}+\mu_{B}^{\text {cont }} \theta_{2}+\frac{1}{2} \theta_{1}^{2} \sigma_{A}^{2}+\frac{1}{2} \theta_{2}^{2} \sigma_{B}^{2}+\theta_{1} \theta_{2} \rho \sigma_{A} \sigma_{B}\right.} \\
& \left.\quad+\lambda_{A}^{\text {cont }, \text { cont }}\left(e^{\theta_{1} \log \left(1+L_{A}\right)}-1\right)+\lambda_{B}^{\text {cont }, \text { cont }}\left(e^{\theta_{2} \log \left(1+L_{B}\right)}-1\right)-\lambda^{\text {cont }, \text { calm }}\right] T \\
& -\frac{K_{1}+Z}{2} T+\log \left(\left(2 e^{\theta_{3}} K_{3}+K_{1}+Z\right)\left(e^{Z T}-1\right)+2 Z\right)-\log (2 Z)+2 \pi i n \\
& +p\left[\log \left[\left(-2 e^{-\theta_{3}} K_{2}-K_{1}+Z\right)\left(e^{Z T}-1\right)+2 Z\right]-\log \left[\left(2 e^{\theta_{3}} K_{3}+K_{1}+Z\right)\left(e^{Z T}-1\right)+2 Z\right]\right] .
\end{aligned}
$$


The indicator variable $p$ takes only the two values 0 and 1 almost surely. For $p=1$, the function $\chi$ simplifies to

$$
\begin{aligned}
& \chi\left(\theta_{1}, \theta_{2}, \theta_{3}, T, 1\right) \\
= & {\left[\mu_{A}^{\text {cont }} \theta_{1}+\mu_{B}^{\text {cont }} \theta_{2}+\frac{1}{2} \theta_{1}^{2} \sigma_{A}^{2}+\frac{1}{2} \theta_{2}^{2} \sigma_{B}^{2}+\theta_{1} \theta_{2} \rho \sigma_{A} \sigma_{B}\right.} \\
& \left.\quad+\lambda_{A}^{\text {cont }, \text { cont }}\left(e^{\theta_{1} \log \left(1+L_{A}\right)}-1\right)+\lambda_{B}^{\text {cont }, \text { cont }}\left(e^{\theta_{2} \log \left(1+L_{B}\right)}-1\right)-\lambda^{\text {cont }, \text { calm }}-\frac{K_{1}+Z}{2}\right] T \\
& \quad+\log \left(\left(-2 e^{-\theta_{3}} K_{2}-K_{1}+Z\right)\left(e^{Z T}-1\right)+2 Z\right)-\log (2 Z)+2 \pi i n .
\end{aligned}
$$

On the other hand, for $p=0$, the function $\chi$ simplifies to

$$
\begin{aligned}
& \chi\left(\theta_{1}, \theta_{2}, \theta_{3}, T, 0\right) \\
= & {\left[\mu_{A}^{c o n t} \theta_{1}+\mu_{B}^{\text {cont }} \theta_{2}+\frac{1}{2} \theta_{1}^{2} \sigma_{A}^{2}+\frac{1}{2} \theta_{2}^{2} \sigma_{B}^{2}+\theta_{1} \theta_{2} \rho \sigma_{A} \sigma_{B}\right.} \\
& \left.\quad+\lambda_{A}^{\text {cont }, \text { cont }}\left(e^{\theta_{1} \log \left(1+L_{A}\right)}-1\right)+\lambda_{B}^{\text {cont,cont }}\left(e^{\theta_{2} \log \left(1+L_{B}\right)}-1\right)-\lambda^{\text {cont }, \text { calm }}-\frac{K_{1}+Z}{2}\right] T \\
& +\log \left(\left(2 e^{\theta_{3}} K_{3}+K_{1}+Z\right)\left(e^{Z T}-1\right)+2 Z\right)-\log (2 Z)+2 \pi i n .
\end{aligned}
$$

Therefore, $\chi$ can be rewritten as

$$
\chi\left(\theta_{1}, \theta_{2}, \theta_{3}, T, p\right)=\left(\frac{M_{1}(\theta)}{2}-\frac{Z(\theta)}{2}\right) T+\log \left(e^{Z(\theta) T}+M_{2}(\theta, p)\right)+M_{3}(\theta, p)+2 \pi i n
$$

where $M_{1}, M_{2}, M_{3}$ are defined as follows:

$$
\begin{aligned}
M_{1}(\theta)= & 2\left(\mu_{A}^{\text {cont }} \theta_{1}+\mu_{B}^{\text {cont }} \theta_{2}+\frac{1}{2} \theta_{1}^{2} \sigma_{A}^{2}+\frac{1}{2} \theta_{2}^{2} \sigma_{B}^{2}+\theta_{1} \theta_{2} \rho \sigma_{A} \sigma_{B}\right. \\
& \left.\quad+\lambda_{A}^{\text {cont }, \text { cont }}\left(e^{\theta_{1} \log \left(1+L_{A}\right)}-1\right)+\lambda_{B}^{\text {cont }, \text { cont }}\left(e^{\theta_{2} \log \left(1+L_{B}\right)}-1\right)-\lambda^{\text {cont }, \text { calm }}-\frac{K_{1}(\theta)}{2}\right) \\
= & \left(\mu_{A}^{\text {calm }}+\mu_{A}^{\text {cont }}\right) \theta_{1}+\left(\mu_{B}^{\text {calm }}+\mu_{B}^{\text {cont }}\right) \theta_{2}+\theta_{1}^{2} \sigma_{A}^{2}+\theta_{2}^{2} \sigma_{B}^{2}+2 \theta_{1} \theta_{2} \rho \sigma_{A} \sigma_{B} \\
& \quad+\lambda_{A}^{c a l m, \text { calm }}\left(e^{\theta_{1} \log \left(1+L_{A}\right)}-1\right)+\lambda_{B}^{\text {calm,calm }}\left(e^{\theta_{2} \log \left(1+L_{B}\right)}-1\right) \\
& \quad+\lambda_{A}^{\text {cont }, \text { cont }}\left(e^{\theta_{1} \log \left(1+L_{A}\right)}-1\right)+\lambda_{B}^{\text {cont }, \text { cont }}\left(e^{\theta_{2} \log \left(1+L_{B}\right)}-1\right) \\
& \quad-\lambda_{A}^{\text {calm,cont }}-\lambda_{B}^{\text {calm,cont }}-\lambda^{\text {cont,calm }} \\
M_{2}(\theta, p)= & \frac{2 Z(\theta)}{-2 e^{-\theta_{3}} K_{2}(\theta)-K_{1}(\theta)+Z(\theta)} \cdot p+\frac{2 Z(\theta)}{2 e^{\theta_{3}} K_{3}+K_{1}(\theta)+Z(\theta)} \cdot(1-p)-1 \\
M_{3}(\theta, p)= & p \log \left(-2 e^{-\theta_{3}} K_{2}(\theta)-K_{1}(\theta)+Z(\theta)\right)+(1-p) \log \left(2 e^{\theta_{3}} K_{3}+K_{1}(\theta)+Z(\theta)\right)-\log (2 Z(\theta))
\end{aligned}
$$


The functions $M_{1}, M_{2}, M_{3}$ do not depend on $T$. Therefore, the integral in Lemma 9 can be computed as the sum of two integrals whose integrand is an exponential-affine function of $t$ :

$$
\begin{aligned}
I & =\int_{0}^{\infty} e^{-\delta t+\chi\left(\theta_{1}, \theta_{2}, \theta_{3}, t, p\right)} d t=\int_{0}^{\infty} e^{-\delta t+\left(\frac{M_{1}}{2}-\frac{Z}{2}\right) t+\log \left(e^{Z t}+M_{2}\right)+M_{3}+2 \pi i n} d t \\
& =e^{M_{3}} \int_{0}^{\infty} e^{\left(\frac{M_{1}}{2}-\frac{Z}{2}-\delta\right) t}\left(e^{Z t}+M_{2}\right) d t=e^{M_{3}}\left(\int_{0}^{\infty} e^{\left(\frac{M_{1}}{2}+\frac{Z}{2}-\delta\right) t} d t+M_{2} \int_{0}^{\infty} e^{\left(\frac{M_{1}}{2}-\frac{Z}{2}-\delta\right) t} d t\right) \\
& =e^{M_{3}}\left(-\frac{1}{\frac{M_{1}}{2}+\frac{Z}{2}-\delta}-\frac{M_{2}}{\frac{M_{1}}{2}-\frac{Z}{2}-\delta}\right) \\
& =-\frac{1}{\left(1+M_{2}\right)\left(\frac{M_{1}}{2}+\frac{Z}{2}-\delta\right)}-\frac{M_{2}}{\left(1+M_{2}\right)\left(\frac{M_{1}}{2}-\frac{Z}{2}-\delta\right)}
\end{aligned}
$$

Equality (10) holds although $\frac{M_{1}+Z}{2}-\delta$ and $\frac{M_{1}-Z}{2}-\delta$ are complex numbers because the respective integration rules also apply in complex analysis. Equality (11) holds because $e^{M_{3}(\theta, p)}=\frac{1}{1+M_{2}(\theta, p)}$ for $p=0$ as well as for $p=1$. Altogether, this completes the proof of Lemma 9 .

Now, evaluating the functions $M_{1}(\theta)$ and $M_{2}(\theta, p)$ at

$$
\left(\theta_{1}, \theta_{2}, \theta_{3}, p\right)=\left(\alpha_{1}-\frac{\gamma}{2}-i v, \alpha_{2}-\frac{\gamma}{2}+i v, 0, p_{0}\right)
$$

gives the formula for the price-dividend ratio of Proposition 3.

\section{Proof of Proposition 4}

For $i=A, B$, Ito's Lemma gives

$$
\begin{aligned}
d P_{i, t}= & \frac{\partial P_{i, t}}{\partial D_{A, t}} d D_{A, t}^{d i f f}+\frac{\partial P_{i, t}}{\partial D_{B, t}} d D_{B, t}^{d i f f}+\frac{1}{2}\left(\frac{\partial^{2} P_{i, t}}{\partial D_{A, t}^{2}} d D_{A, t}^{2}+\frac{\partial^{2} P_{i, t}}{\partial D_{B, t}^{2}} d D_{B, t}^{2}+2 \frac{\partial^{2} P_{i, t}}{\partial D_{A, t} \partial D_{B, t}} d D_{A, t} d B_{B, t}\right) \\
& +\sum_{j=1,2,3}\left(P_{i, t}-P_{i, t-}\right)\left[d N_{t}^{A, j}+d N_{t}^{B, j}\right]+\left(P_{i, t}-P_{i, t-}\right) d N_{t}^{4}
\end{aligned}
$$

The dividend processes follow

$$
\begin{aligned}
& \frac{d D_{A, t}}{D_{A, t-}}=\left(\mu_{A}+\frac{1}{2} \sigma_{A}^{2}\right) d t+\sigma_{A} d W_{t}^{A}+L_{A} \sum_{j=1,2,3} d N_{t}^{A, j} \\
& \frac{d D_{B, t}}{D_{B, t-}}=\left(\mu_{B}+\frac{1}{2} \sigma_{B}^{2}\right) d t+\sigma_{B} d W_{t}^{B}+L_{B} \sum_{j=1,2,3} d N_{t}^{B, j}
\end{aligned}
$$

According to Proposition 3, the price of asset $i$ which pays the dividend $D_{A}^{\alpha_{1}} D_{B}^{\alpha_{2}}$ is given by

$$
P_{i, t}=\int_{-\infty}^{\infty} I\left(s_{t}, v\right) D_{i, t} J\left(v, p_{t}, \alpha_{1}, \alpha_{2}\right) d v
$$


where

$$
I(s, v)=\left(\frac{1}{\sqrt{s(1-s)}}\right)^{\gamma}\left(\frac{1-s}{s}\right)^{i v}=\left(D_{A}+D_{B}\right)^{\gamma} D_{A}^{-\frac{\gamma}{2}-i v} D_{B}^{-\frac{\gamma}{2}+i v}
$$

and $J$ does not depend on $D_{A}$ or $D_{B}$ at all. Computing the partial derivatives of $P_{i, t}$ thus breaks down to computing the partial derivatives of $I$ (due to a standard dominated convergence argument, differential and integral signs can be interchanged here if all involved integrals exist). The first-order derivatives of $I$ fulfil

$$
\begin{aligned}
\frac{\partial I}{\partial D_{A}} D_{A} & =I \cdot\left[\gamma s-\frac{\gamma}{2}-i v\right] \\
\frac{\partial I}{\partial D_{B}} D_{B} & =I \cdot\left[\gamma(1-s)-\frac{\gamma}{2}+i v\right] \\
\frac{\partial\left[I D_{A}\right]}{\partial D_{A}}=\frac{\partial I}{\partial D_{A}} D_{A}+I & =I \cdot\left[\gamma s-\frac{\gamma}{2}-i v+1\right] \\
\frac{\partial\left[I D_{B}\right]}{\partial D_{B}}=\frac{\partial I}{\partial D_{B}} D_{B}+I & =I \cdot\left[\gamma(1-s)-\frac{\gamma}{2}+i v+1\right]
\end{aligned}
$$

The exposure of asset $i$ to diffusion risk $j$ is defined as

$$
\sigma_{i, j, t}=\frac{\frac{\partial P_{i, t}}{\partial D_{j, t}} D_{j, t}}{P_{i, t}} \sigma_{j}
$$

Remember the abbreviation $\left(\theta_{1}, \theta_{2}\right)=\left(\alpha_{1}-\frac{\gamma}{2}-i v, \alpha_{2}-\frac{\gamma}{2}+i v\right)$ where $\left(\alpha_{1}, \alpha_{2}\right)=(1,0)$ for asset $A$ and $(0,1)$ for asset B. Plugging in the derivatives and eliminating $D_{A, t}$ and $D_{B, t}$ whenever they are redundant leads to the general form for the diffusion exposures given in Proposition 4 . The exposure of asset $i$ to one of the seven jump risk factors is defined as

$$
\frac{P_{i, t}-P_{i, t-}}{P_{i, t-}} .
$$

The exposure to jumps from the contagion state to the calm state which do not induce any dividend losses (i.e. $L_{i, 4}$ ) follows from this definition directly.

In order to analyze the jumps which imply a loss in one of the dividends (i.e. type $j=1,2,3$ ), let the dividend process which exhibits the jump be encoded by $\left(\tilde{\alpha}_{1}, \tilde{\alpha}_{2}\right)$ and let the asset whose price exposure is to be investigated be encoded by $\left(\alpha_{1}, \alpha_{2}\right)$. Then each jump exposure can be written in the form

$$
L_{\left(\alpha_{1}, \alpha_{2}\right),\left(\tilde{\alpha}_{1}, \tilde{\alpha}_{2}\right), j}=\left(1+L_{A}\right)^{\alpha_{1} \tilde{\alpha}_{1}}\left(1+L_{B}\right)^{\alpha_{2} \tilde{\alpha}_{2}} \frac{\int_{-\infty}^{\infty} I\left(s_{t}, v\right) J\left(v, p_{t}, \alpha_{1}, \alpha_{2}\right) d v}{\int_{-\infty}^{\infty} I\left(s_{t-}, v\right) J\left(v, p_{t-}, \alpha_{1}, \alpha_{2}\right) d v}-1
$$

If dividends of asset $A$ jump, the dividend share decreases to $s_{t}=s_{t-} \frac{1+L_{A}}{1+L_{A} s_{t-}}$. If dividends of asset $B$ jump, it increases to $s_{t}=s_{t-} \frac{1}{1+L_{B}\left(1-s_{t-}\right)}$. Putting all details together leads to the 
following jump exposures:

$$
\begin{aligned}
L_{i, A, 1}(s, p) & =\left(1+L_{A}\right)^{\alpha_{1}} \frac{\int_{-\infty}^{\infty} I\left(s \frac{1+L_{A}}{1+s L_{A}}, v\right) J\left(v, 1, \alpha_{1}, \alpha_{2}\right) d v}{\int_{-\infty}^{\infty} I(s, v) J\left(v, 1, \alpha_{1}, \alpha_{2}\right) d v}-1 \\
L_{i, B, 1}(s, p) & =\left(1+L_{B}\right)^{\alpha_{2}} \frac{\int_{-\infty}^{\infty} I\left(s \frac{1}{1+(1-s) L_{B}}, v\right) J\left(v, 1, \alpha_{1}, \alpha_{2}\right) d v}{\int_{-\infty}^{\infty} I(s, v) J\left(v, 1, \alpha_{1}, \alpha_{2}\right) d v}-1 \\
L_{i, A, 2}(s, p) & =\left(1+L_{A}\right)^{\alpha_{1}} \frac{\int_{-\infty}^{\infty} I\left(s \frac{1+L_{A}}{1+s L_{A}}, v\right) J\left(v, 0, \alpha_{1}, \alpha_{2}\right) d v}{\int_{-\infty}^{\infty} I(s, v) J\left(v, 1, \alpha_{1}, \alpha_{2}\right) d v}-1 \\
L_{i, B, 2}(s, p) & =\left(1+L_{B}\right)^{\alpha_{2}} \frac{\int_{-\infty}^{\infty} I\left(s \frac{1}{1+(1-s) L_{B}}, v\right) J\left(v, 0, \alpha_{1}, \alpha_{2}\right) d v}{\int_{-\infty}^{\infty} I(s, v) J\left(v, 1, \alpha_{1}, \alpha_{2}\right) d v}-1 \\
L_{i, A, 3}(s, p) & =\left(1+L_{A}\right)^{\alpha_{1}} \frac{\int_{-\infty}^{\infty} I\left(s \frac{1+L_{A}}{1+s L_{A}}, v\right) J\left(v, 0, \alpha_{1}, \alpha_{2}\right) d v}{\int_{-\infty}^{\infty} I(s, v) J\left(v, 0, \alpha_{1}, \alpha_{2}\right) d v}-1 \\
L_{i, B, 3}(s, p)= & \left(1+L_{B}\right)^{\alpha_{2}} \frac{\int_{-\infty}^{\infty} I\left(s \frac{1}{1+(1-s) L_{B}}, v\right) J\left(v, 0, \alpha_{1}, \alpha_{2}\right) d v}{\int_{-\infty}^{\infty} I(s, v) J\left(v, 0, \alpha_{1}, \alpha_{2}\right) d v}-1 \\
L_{i, 4}(s, p)= & \frac{\int_{-\infty}^{\infty} I(s, v) J\left(v, 1, \alpha_{1}, \alpha_{2}\right) d v}{\int_{-\infty}^{\infty} I(s, v) J\left(v, 0, \alpha_{1}, \alpha_{2}\right) d v}-1 .
\end{aligned}
$$




\section{References}

Ait-Sahalia, Y., J. Cacho-Diaz, and R. Laeven, 2010, "Modeling Financial Contagion Using Mutually Exciting Jump Processes," Working Paper.

Backus, D., M. Chernov, and I. Martin, 2011, "Disasters Implied by Equity Index Options," Journal of Finance, forthcoming.

Bae, K., A. Karolyi, and R. Stulz, 2003, "A New Approach to Measuring Financial Contagion," Review of Financial Studies, 16(3), 717-763.

Barro, R., 2006, "Rare Disasters and Asset Markets in the Twentieth Century," Quarterly Journal of Economics, 121(3), 823-866.

— , 2009, "Rare Disasters, Asset Prices, and Welfare Costs," American Economic Review, 99(1), 243-264.

Branger, N., C. Schlag, and L. Wu, 2011, "Pricing Two Heterogenous Trees," Journal of Financial and Quantitative Analysis, forthcoming.

Buraschi, A., P. Porchia, and F. Trojani, 2010, "The Cross-Section of Expected Stock Returns: Learning about Distress and Predictability in Heterogeneous Orchards," Working Paper.

Chen, H., and S. Joslin, 2010, "Generalized Transform Analysis of Affine Processes: with an Application to a Model of Time-varying Labor Income Risk," Working Paper.

Claessens, S., and K. Forbes, 2001, International Financial Contagion. Kluwer Academic Publishers, Dordrecht.

Cochrane, J., F. Longstaff, and P. Santa-Clara, 2008, "Two Trees," Review of Financial Studies, 21(1), 347-385.

Duffie, D., J. Pan, and K. Singleton, 2000, "Transform Analysis and Asset Pricing for Affine Jump-Diffusions," Econometrica, 68(6), 1343-1376.

Forbes, K., and R. Rigobon, 2002, "No Contagion, Only Interdependence: Measuring Stock Market Comovements," Journal of Finance, 57(5), 2223-2261.

Gabaix, X., 2010, "Variable Rare Disasters: An Exactly Solved Framework for Ten Puzzles in Macrofinance," Working Paper. 
Liu, J., and J. Pan, 2003, "Dynamic Derivative Strategies," Journal of Financial Economics, 69, 401-430.

Longin, F., and B. Solnik, 2001, "Extreme Correlation of International Equity Markets," Journal of Finance, 56(2), 649-676.

Martin, I., 2009, "The Lucas Orchard," Working Paper.

Naik, V., and M. Lee, 1990, "General equilibrium pricing of options on the market portfolio with discontinuous returns," Review of Financial Studies, 3, 493-521.

Rietz, T., 1988, "The equity premium: A solution," Journal of Monetary Economics, 22, $117-131$.

Wachter, J., 2010, "Can time-varying risk of rare disasters explain aggregate stock market volatility?," Working Paper. 
Benchmark Extension 1 Extension 2 Equal Growth Rates

\begin{tabular}{lcccc}
\hline \hline Panel A: Parameters & & & \\
\hline \hline$\mu_{A}^{\text {calm }}$ & 0.035 & 0.035 & 0.035 & 0.035 \\
$\mu_{A}^{\text {cont }}$ & 0.035 & 0.035 & 0.035 & 0.067 \\
$\mu_{B}^{\text {calm }}$ & 0.035 & 0.035 & 0.035 & 0.035 \\
$\mu_{B}^{\text {cont }}$ & 0.035 & 0.035 & 0.035 & 0.067 \\
\hline$\sigma_{A}$ & 0.08 & 0.08 & 0.08 & 0.08 \\
$\sigma_{B}$ & 0.08 & 0.08 & 0.08 & 0.08 \\
$\rho$ & 0 & 0 & 0 & 0 \\
\hline$L_{A}$ & -0.02 & -0.02 & -0.02 & -0.02 \\
$L_{B}$ & -0.02 & -0.02 & -0.02 & -0.02 \\
\hline$\lambda_{A}^{\text {calm,calm }}$ & 0.2 & 0.2 & 0.0 & 0.2 \\
$\lambda_{A}^{\text {calm,cont }}$ & 0.2 & 0.2 & 0.4 & 0.2 \\
$\lambda_{B}^{\text {calm,calm }}$ & 0.2 & 0.2 & 0.4 & 0.2 \\
$\lambda_{B}^{\text {calm,cont }}$ & 0.2 & 0.2 & 0.0 & 2 \\
\hline$\lambda_{A}^{\text {cont,cont }}$ & 2 & 0.8 & 2 & 2 \\
$\lambda_{B}^{\text {cont,cont }}$ & 2 & 3.2 & 2 & \\
$\lambda^{\text {cont,calm }}$ & 2 & 2 & 2 & 0.03 \\
\hline \hline Panel B: Moments of Dividend Growth & & & 0.03 \\
\hline \hline mean dividend growth A calm & 0.03 & 0.03 & 0.03 & 0.03 \\
mean dividend growth B calm & 0.03 & 0.03 & 0.03 & 0.03 \\
mean dividend growth A cont & 0.00 & 0.02 & 0.03 & 0.081 \\
mean dividend growth B cont & 0.00 & -0.03 & 0.03 & 0.085 \\
\hline dividend volatility A calm & 0.081 & 0.081 & 0.081 & 0.081 \\
dividend volatility B calm & 0.081 & 0.081 & 0.085 & 0.085 \\
dividend volatility A cont & 0.085 & 0.082 & & \\
dividend volatility B cont & 0.085 & 0.088 & 0.035 \\
\hline
\end{tabular}

Table 1: Parameters and Moments

The table gives the parameters (panel A) and the moments (panel B) of the dividends. The first column refers to the benchmark case with identical trees. The last three columns give the parameters and moments if contagion mainly affects asset B ('Extension 1'), if contagion is only induced by asset A ('Extension 2'), and if the dividend growth rates are equal in both states ('Equal Growth Rates'). 

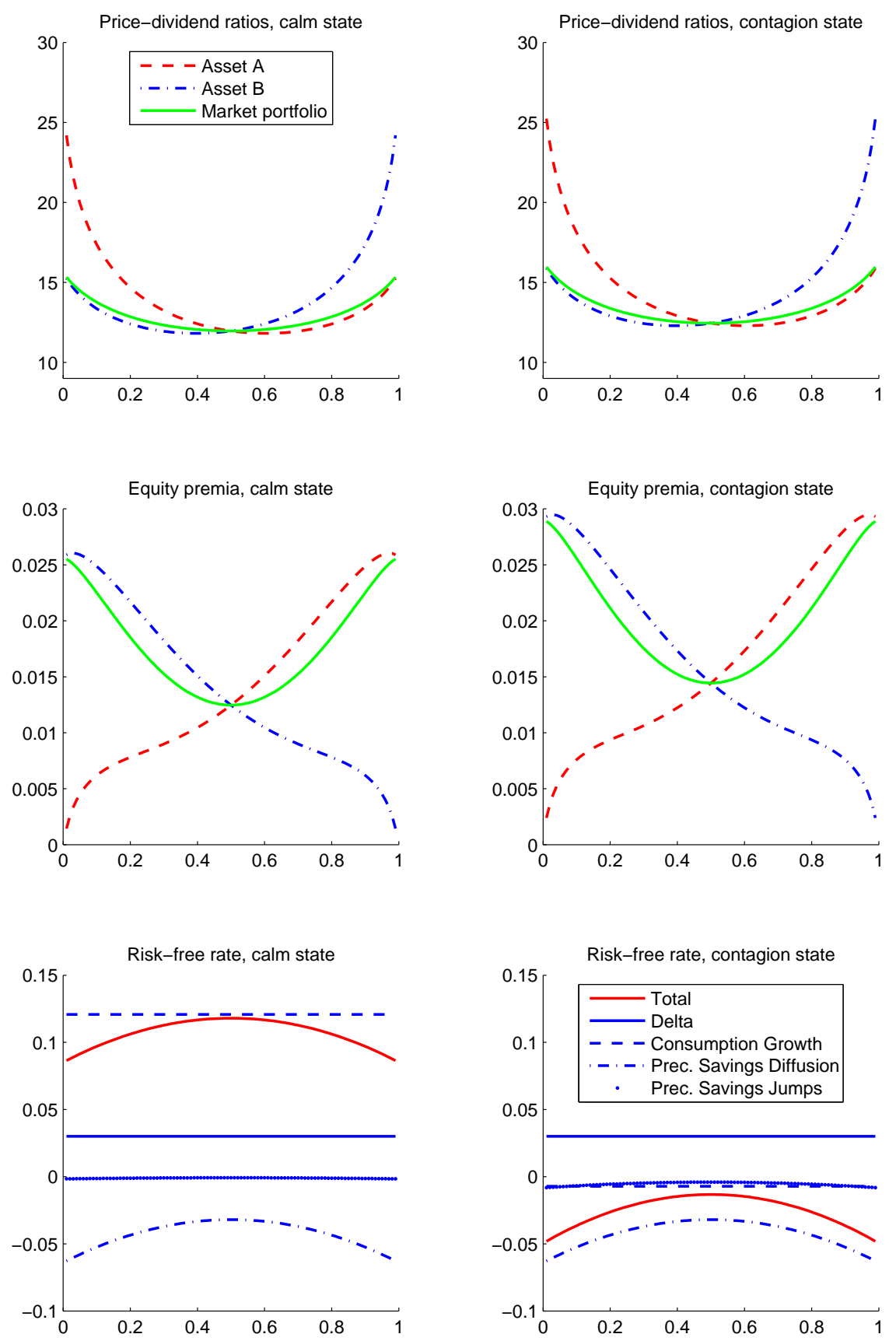

Figure 1: Asset Prices and Returns (Benchmark Parametrization)

The figure shows the price-dividend ratios (upper row), the equity risk premia (middle row) and the risk-free rate (lower row) as a function of the dividend share of asset $\mathrm{A}$. The left column gives the results for the calm state, the right column gives the results for the contagion state. Price-dividend ratios and equity premia are given for asset A (red dashed line), asset B (blue dash-dotted line) and the market (green solid line). The risk-free rate is decomposed into its various components. The parameters for the benchmark case are given in column 'Benchmark' of Table 1. 

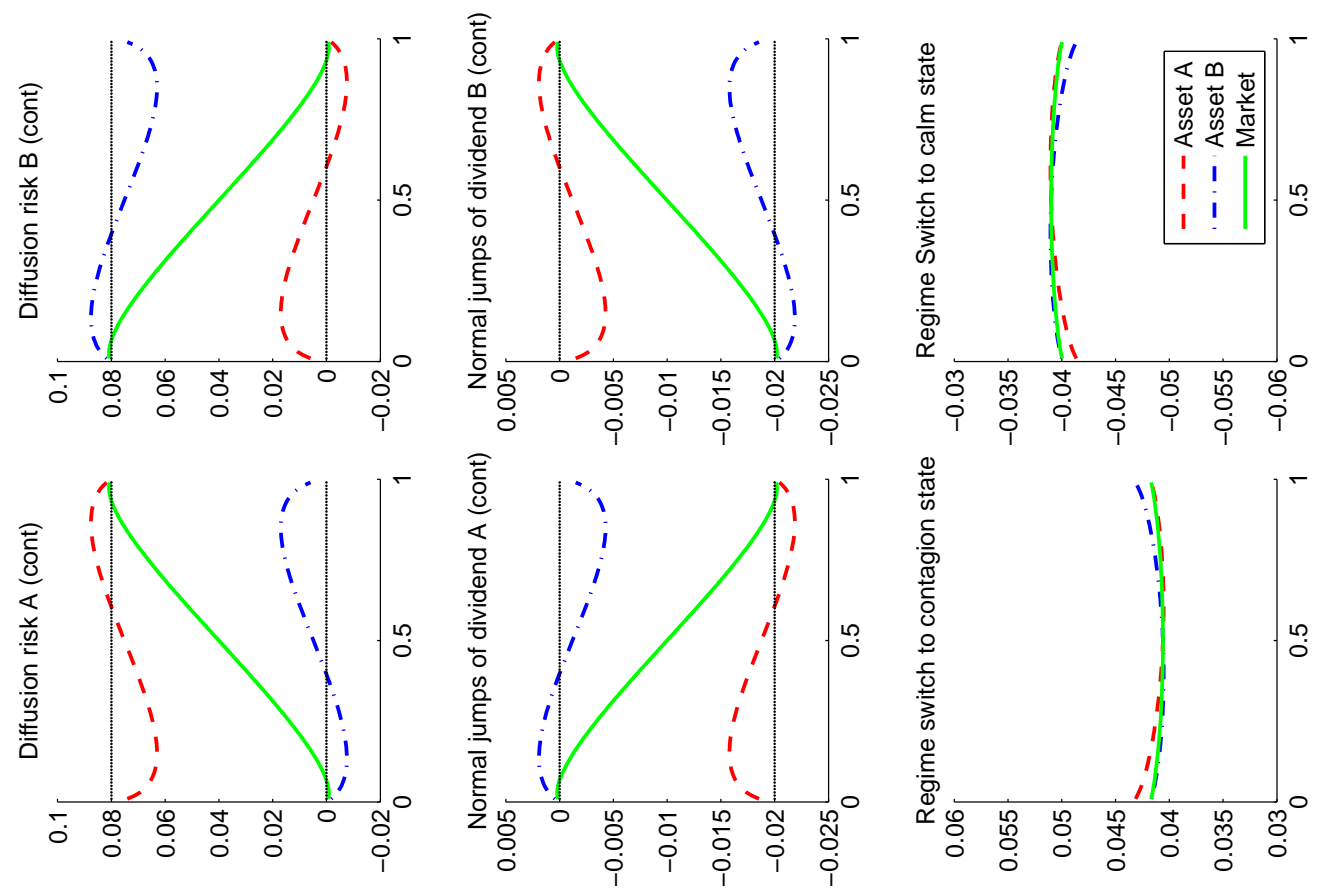

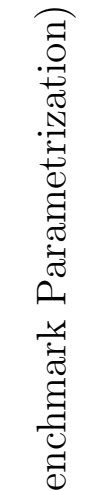
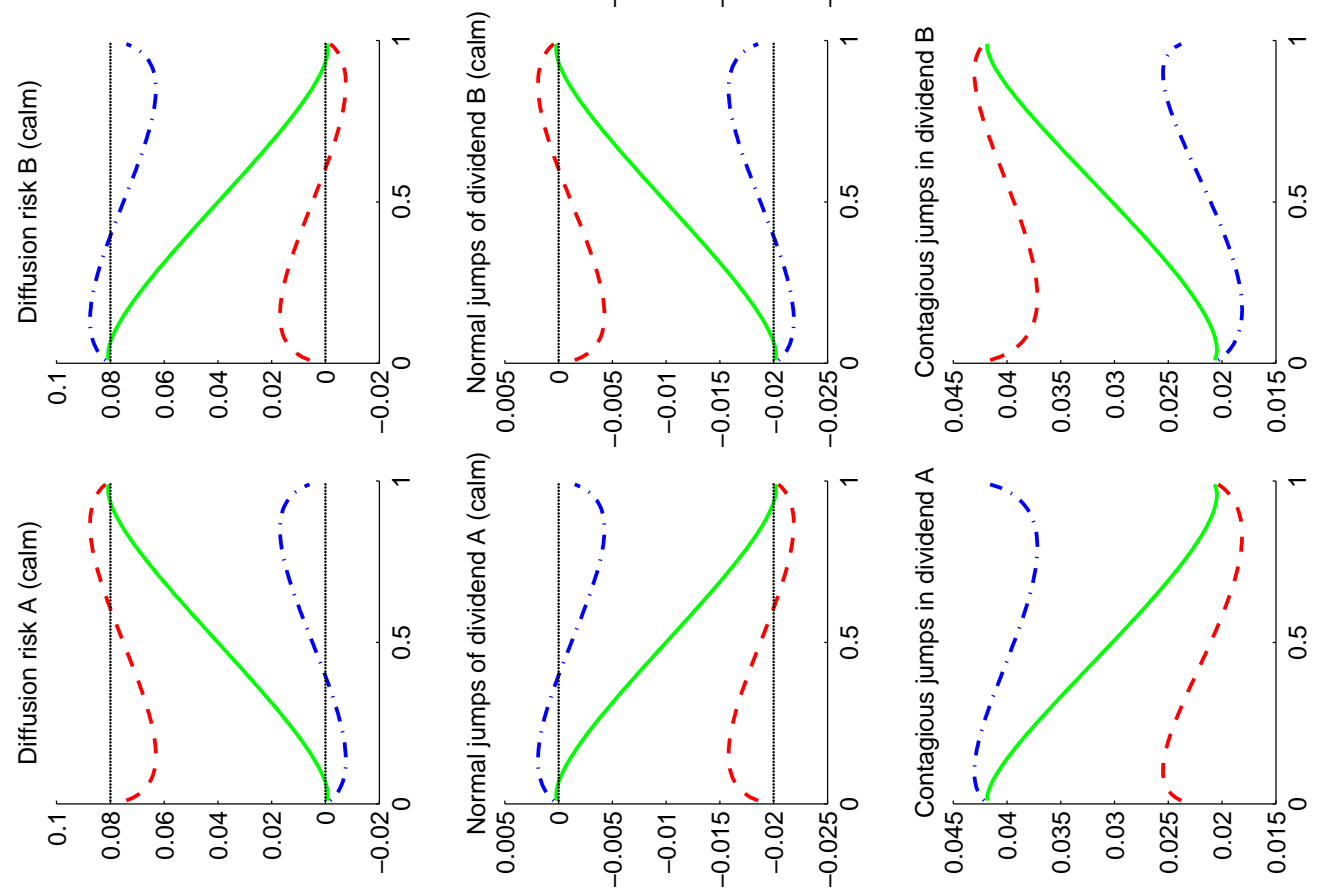

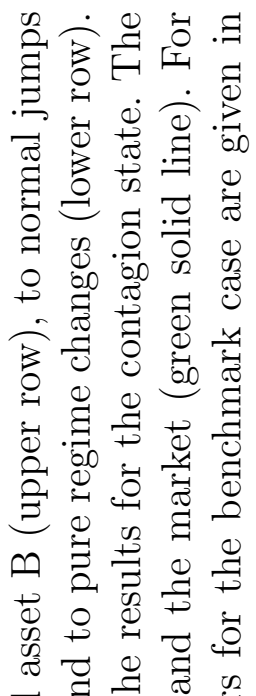

表

สี

$<50.50$

焉焉焉

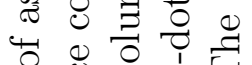

O 0 过

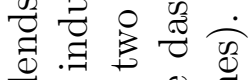

.

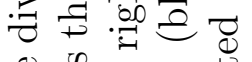

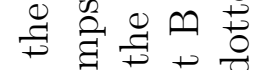

$\exists \Xi \pm$

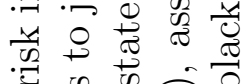

प्र

.

纯

옹

पु

焉

. D

चु है 다

屯.

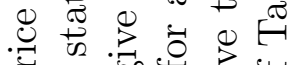

焗

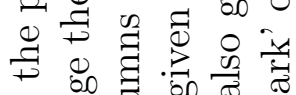

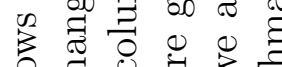

-

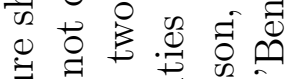

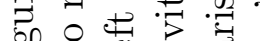

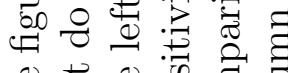

迅莺焉 

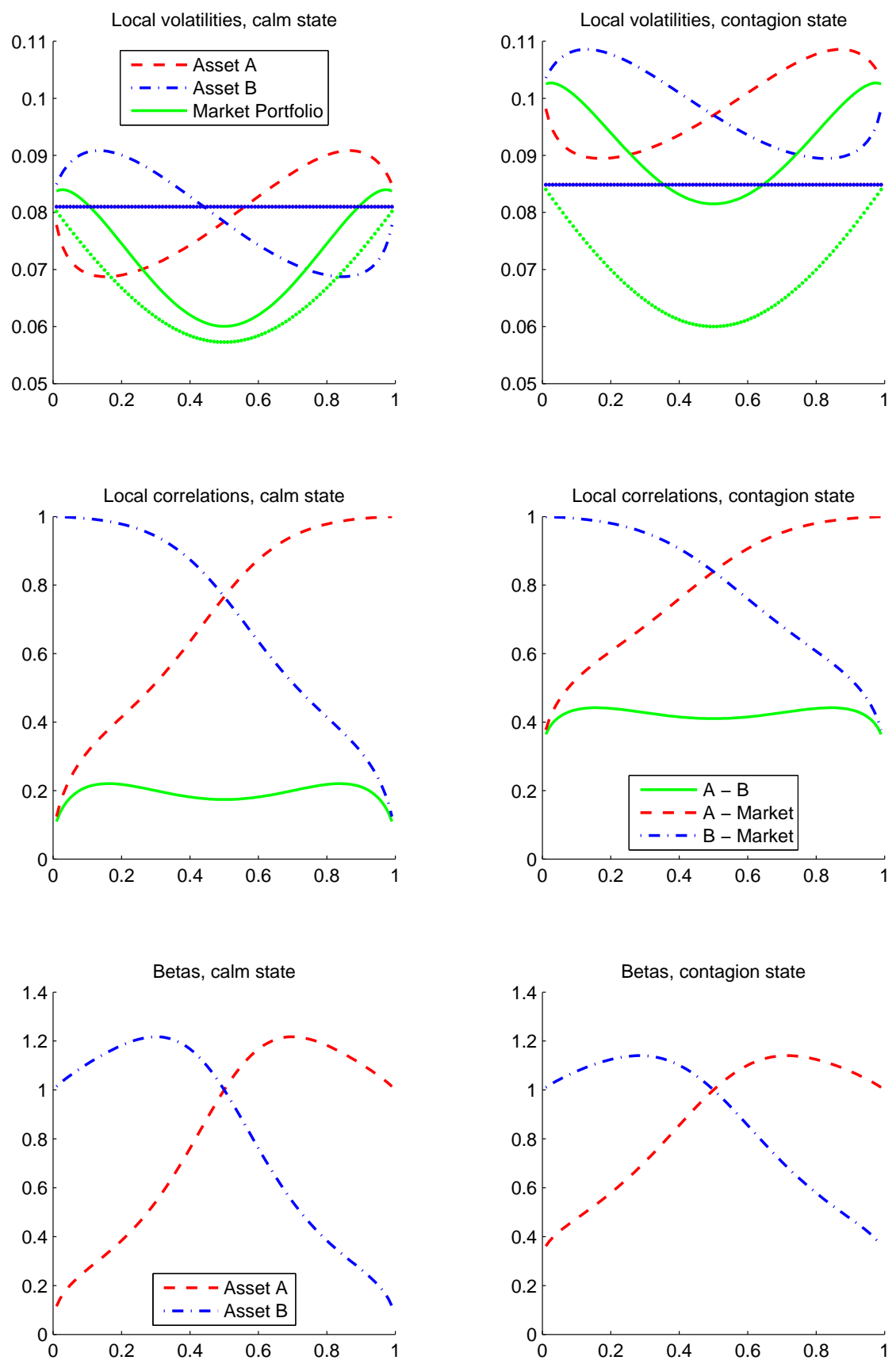

Figure 3: Volatilities and Correlations (Benchmark Parametrization)

The figure shows the local volatilities (upper row), the local correlations (middle row) and the beta with respect to the market (lower row) as a function of the dividend share of asset A. The left column gives the results for the calm state, the right column gives the results for the contagion state. The volatilities are given for asset A (red dashed line), asset B (blue dash-dotted line) and the market (green solid line) and the respective dotted lines mark the dividend and consumption volatilities. The parameters for the benchmark case are given in column 'Benchmark' of Table 1. 

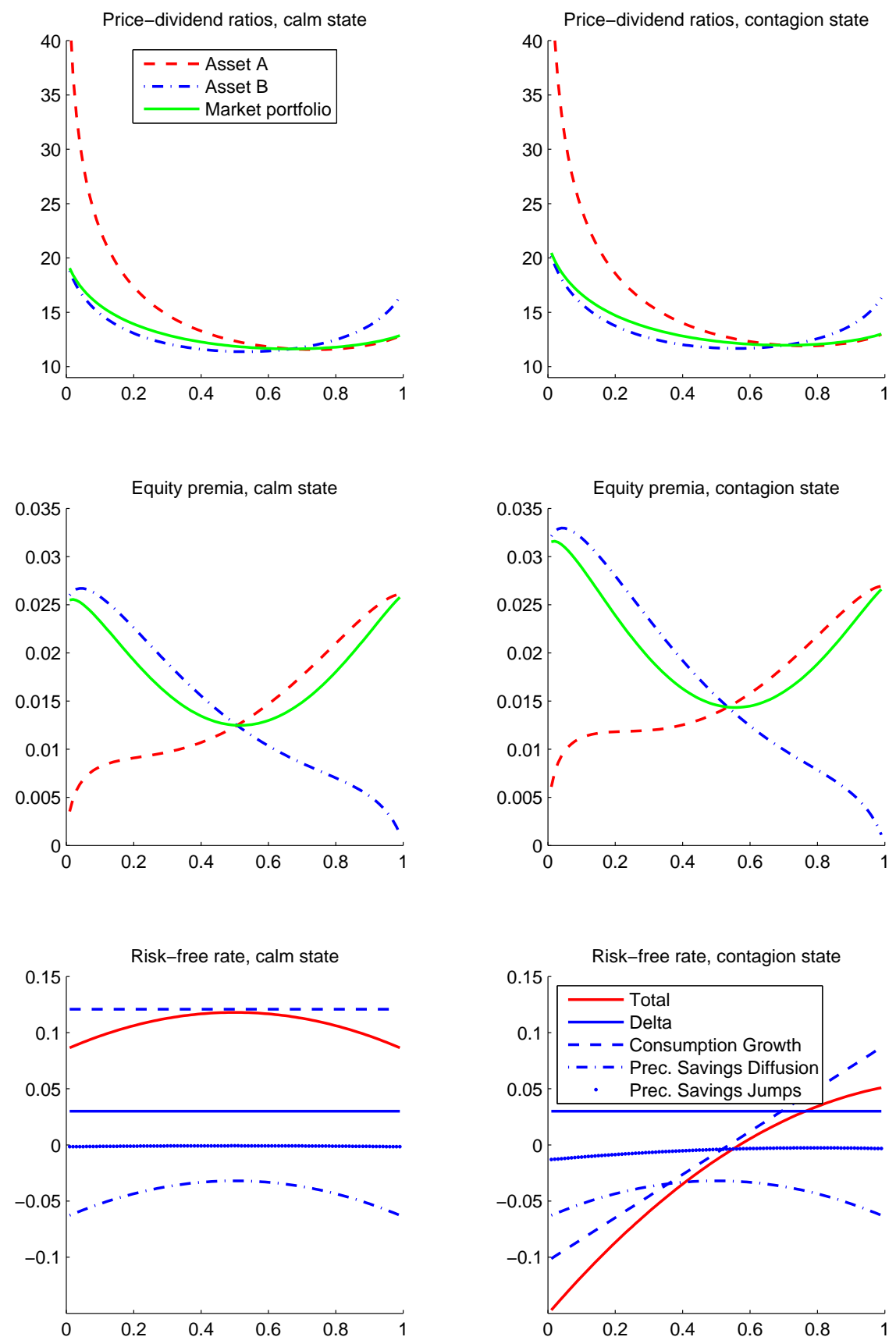

Figure 4: Asset Prices and Returns (Robust versus Excitable Assets)

The figure shows the price-dividend ratios (upper row), the equity risk premia (middle row) and the risk-free rate (lower row) as a function of the dividend share of asset A. The left column gives the results for the calm state, the right column gives the results for the contagion state. Price-dividend ratios and equity premia are given for the robust asset A (red dashed line), the excitable asset B (blue dash-dotted line) and the market (green solid line). The risk-free rate is decomposed into its various components. The parameters are given in column 'Extension 1' of Table 1. 

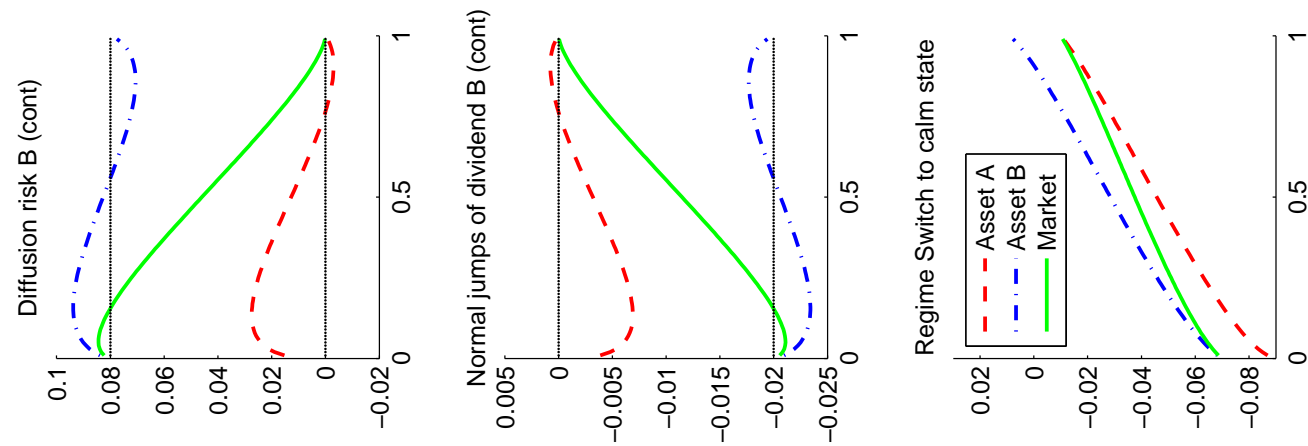

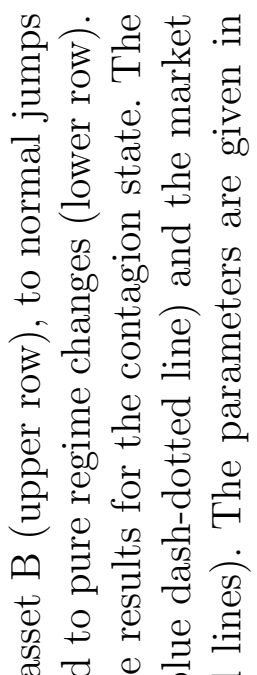
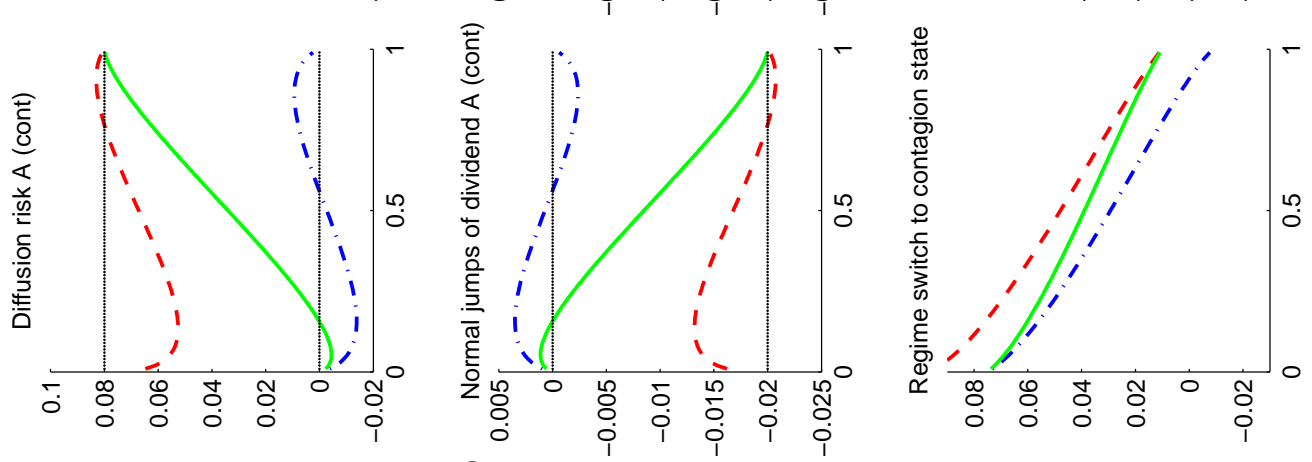

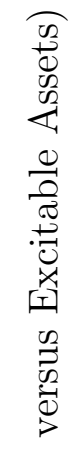

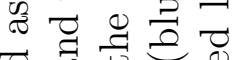

营

$<\cdot \frac{0}{50} \cdot 500$

Ð

की

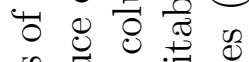

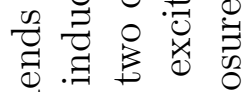

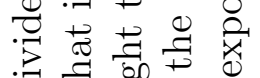
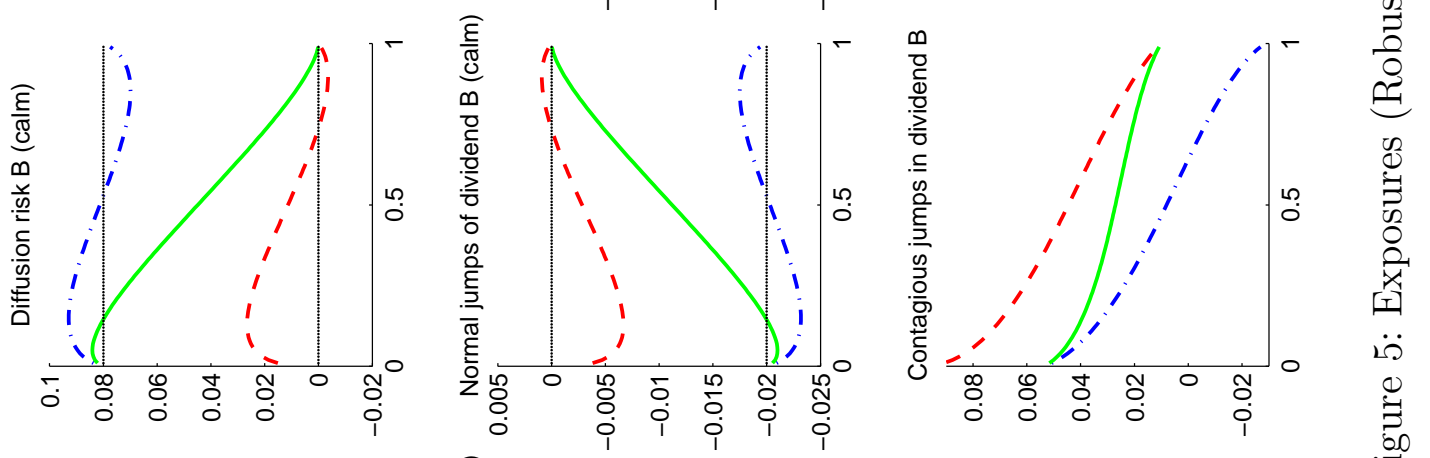

क ज क

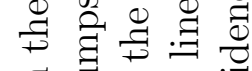

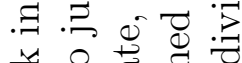

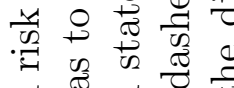

䨌

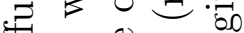

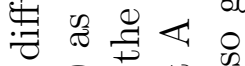

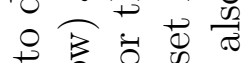

की $0 \begin{array}{lll}0 & 0 & 0\end{array}$
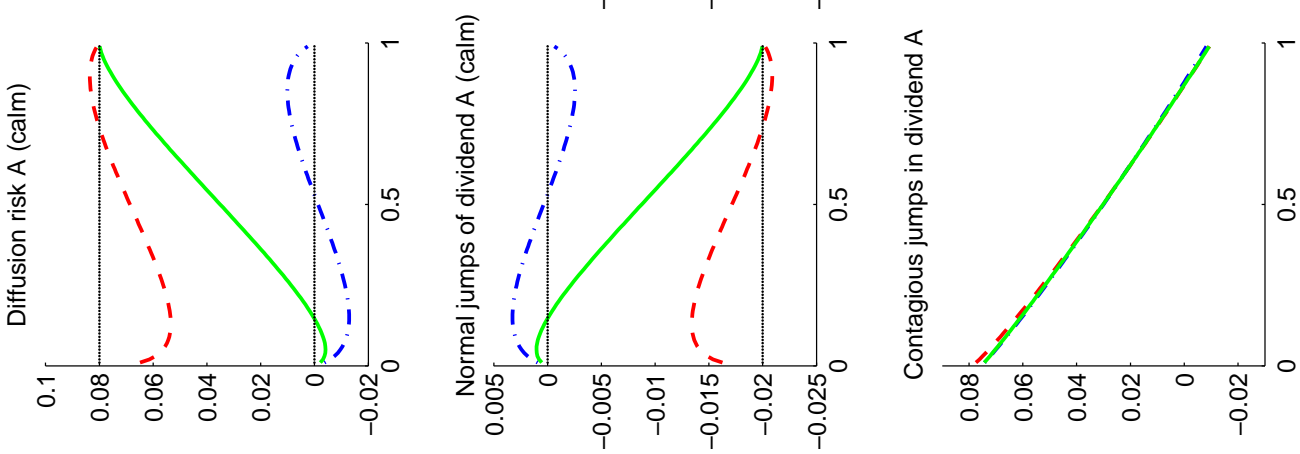

壱芑声苟

:

कृ

○

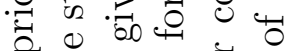

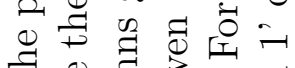

क

范

ज 0 \&

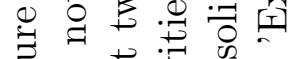

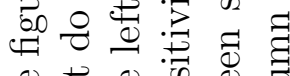

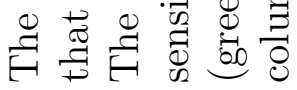




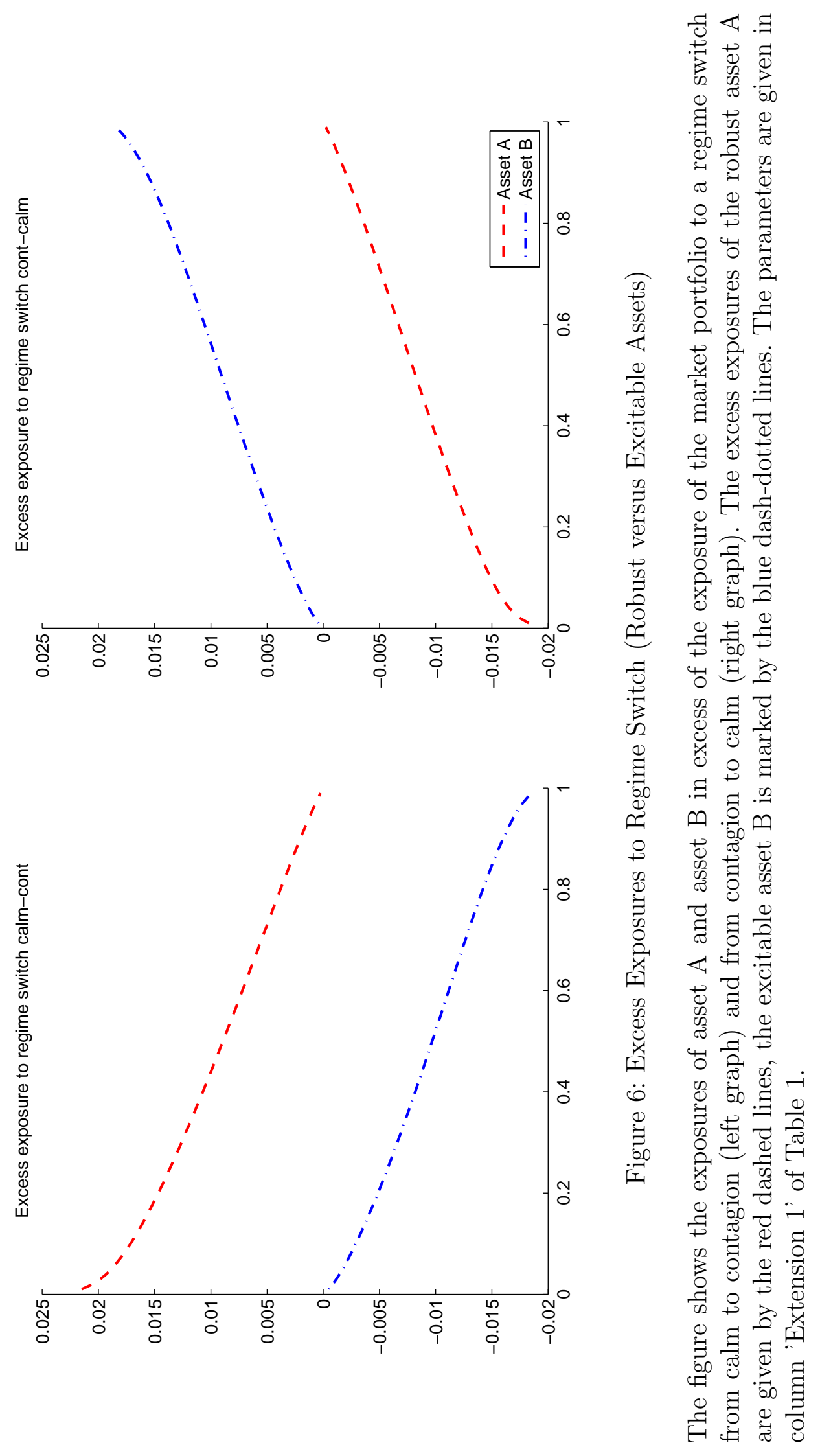



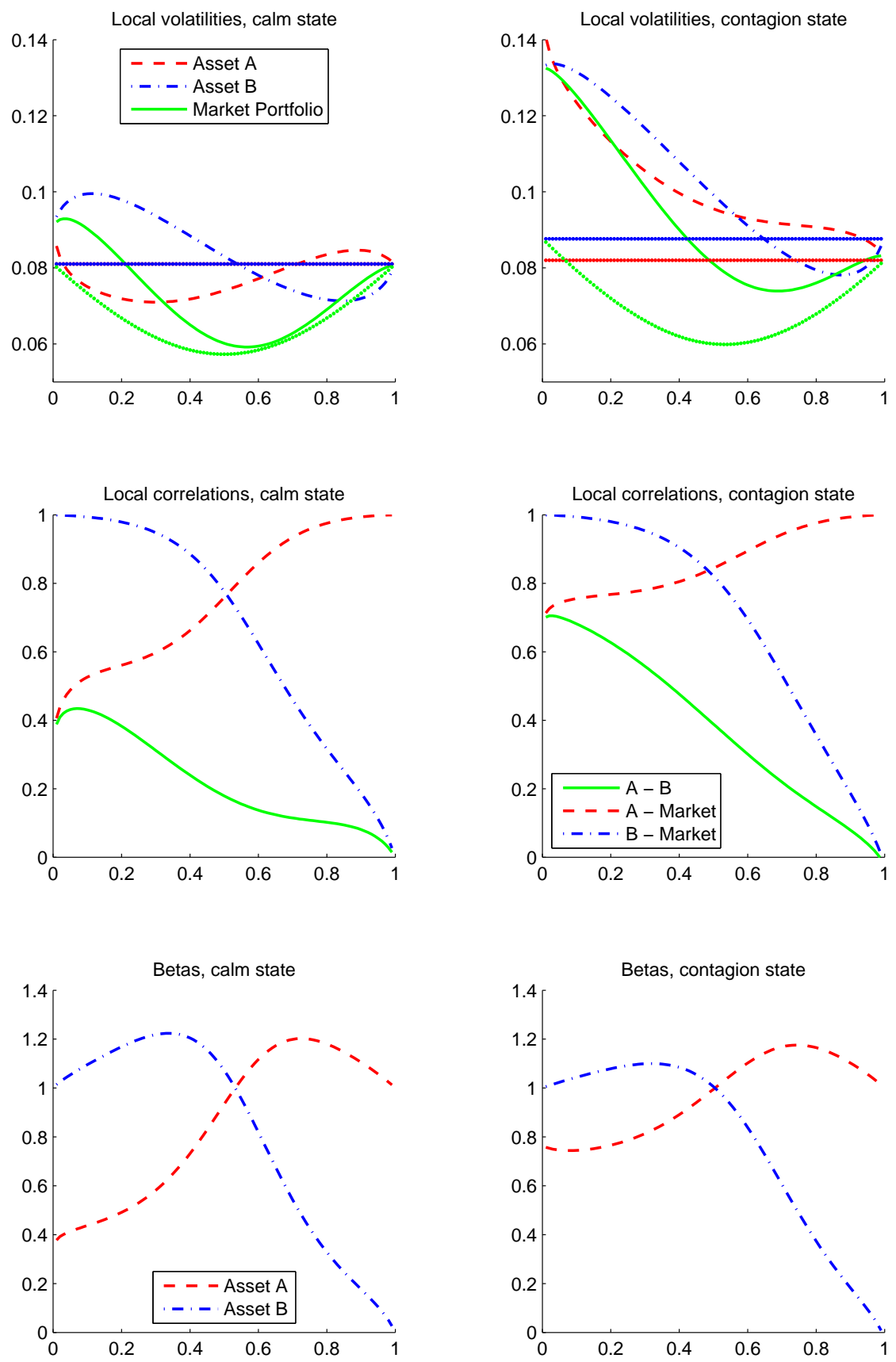

Figure 7: Volatilities and Correlations (Robust versus Excitable Assets)

The figure shows the local volatilities (upper row), the local correlations (middle row) and the beta with respect to the market (lower row) as a function of the dividend share of asset A. The left column gives the results for the calm state, the right column gives the results for the contagion state. The volatilities are given for asset A (red dashed line), asset B (blue dash-dotted line) and the market (green solid line) and the respective dotted lines mark the dividend and consumption volatilities. The parameters are given in column 'Extension 1' of Table 1. 

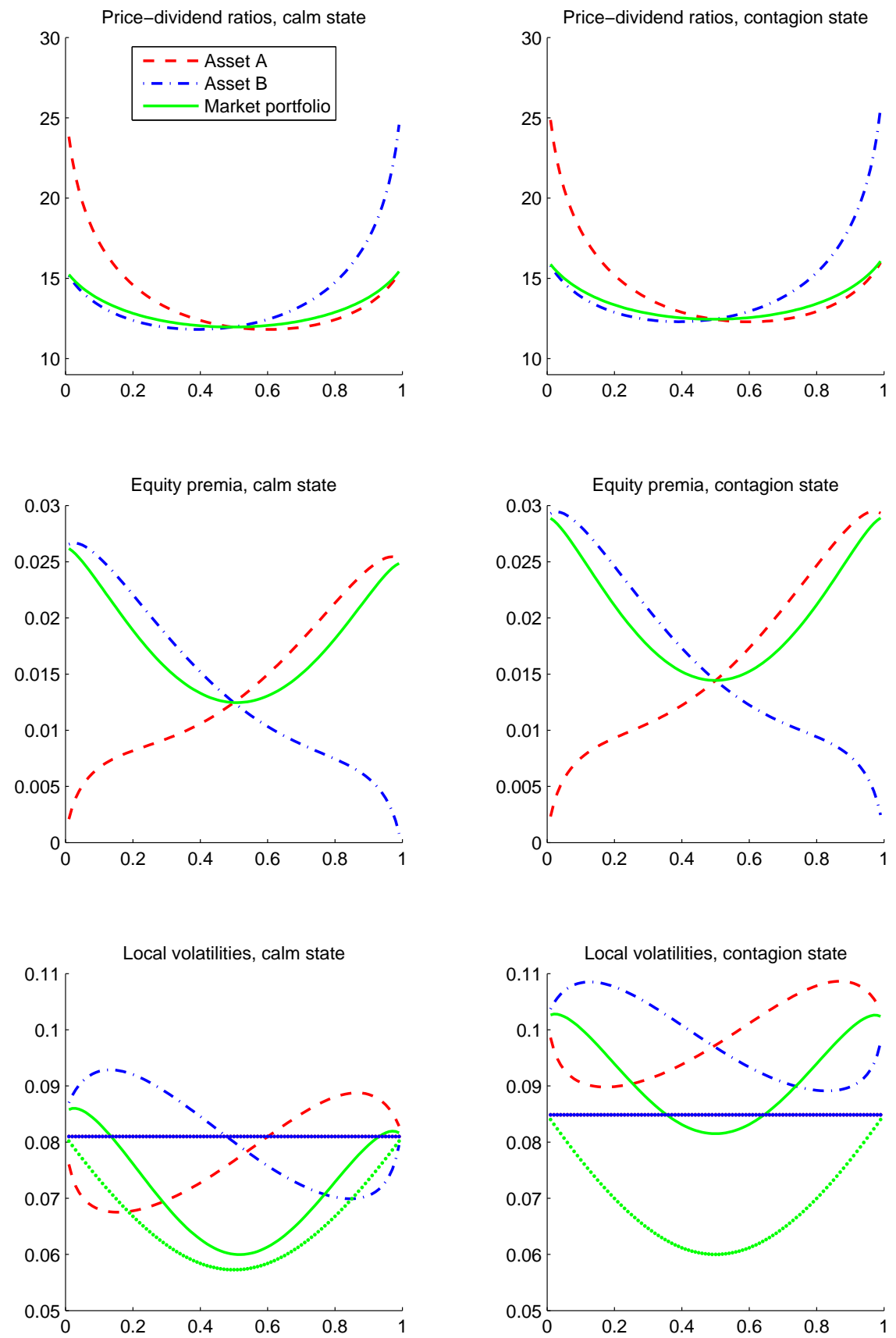

Figure 8: Asset Prices, Equity Premia and Volatilities (Propensity to Induce Contagion)

The figure shows the price-dividend ratios (upper row), the equity risk premia (middle row) and local volatilities (lower row) as a function of the dividend share of asset A. The left column gives the results for the calm state, the right column gives the results for the contagion state. All results are given for the contagion-inducing asset A (red dashed line), the non-inducing asset B (blue dash-dotted line) and the market (green solid line). The parameters are given in column 'Extension 2' of Table 1. 

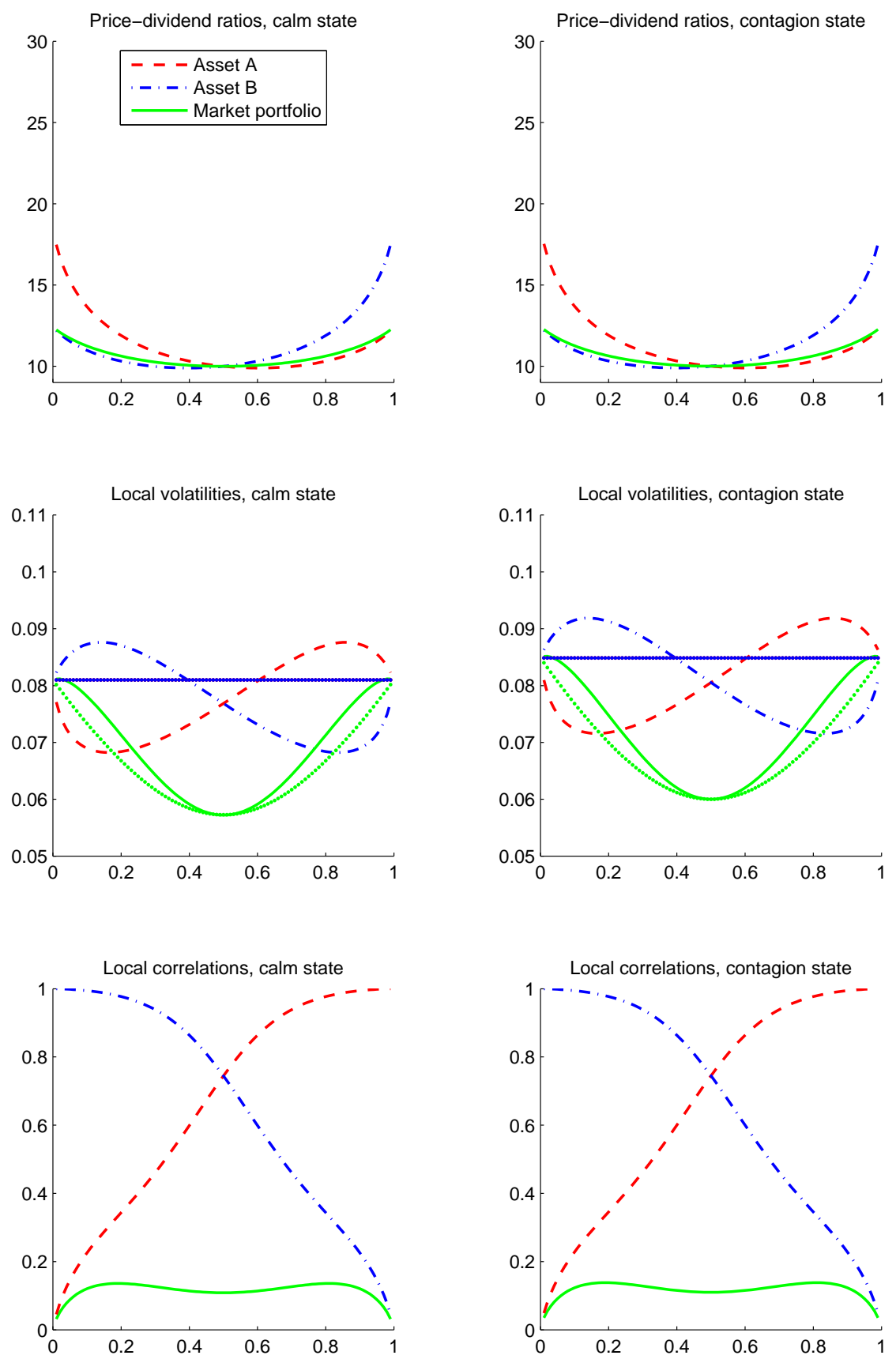

Figure 9: Asset Prices, Volatilities and Correlations (Constant Dividend Growth)

The figure shows the price-dividend ratios (upper row), local volatilities (middle row) and local correlations (lower row) as a function of the dividend share of asset A. The left column gives the results for the calm state, the right column gives the results for the contagion state. The parameters are given in column 'Equal Growth Rates' of Table 1. 

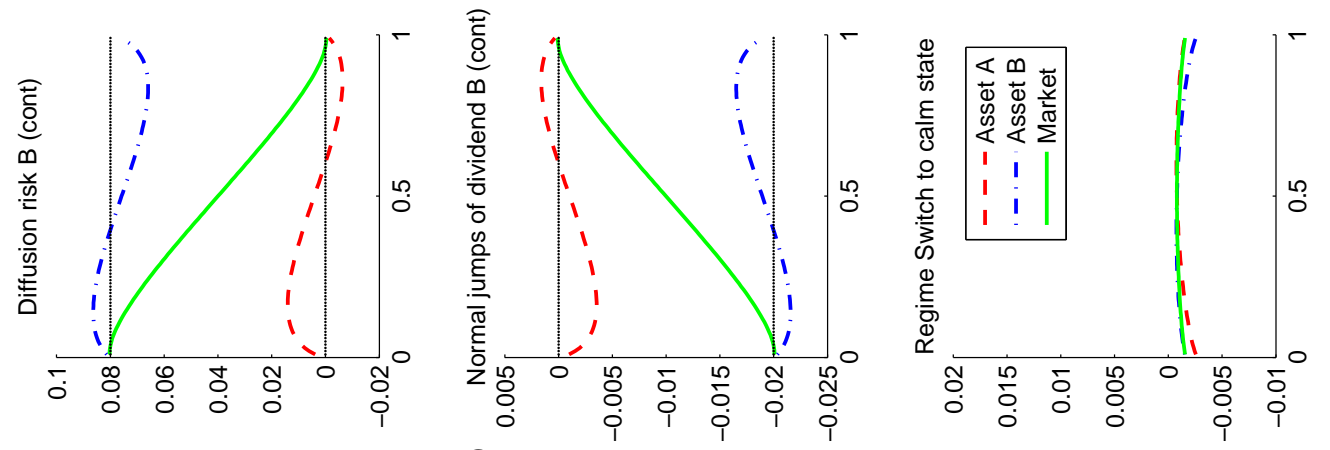

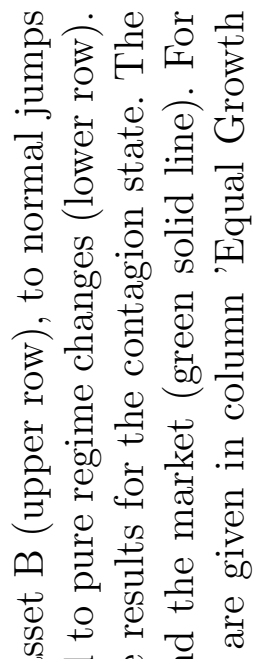
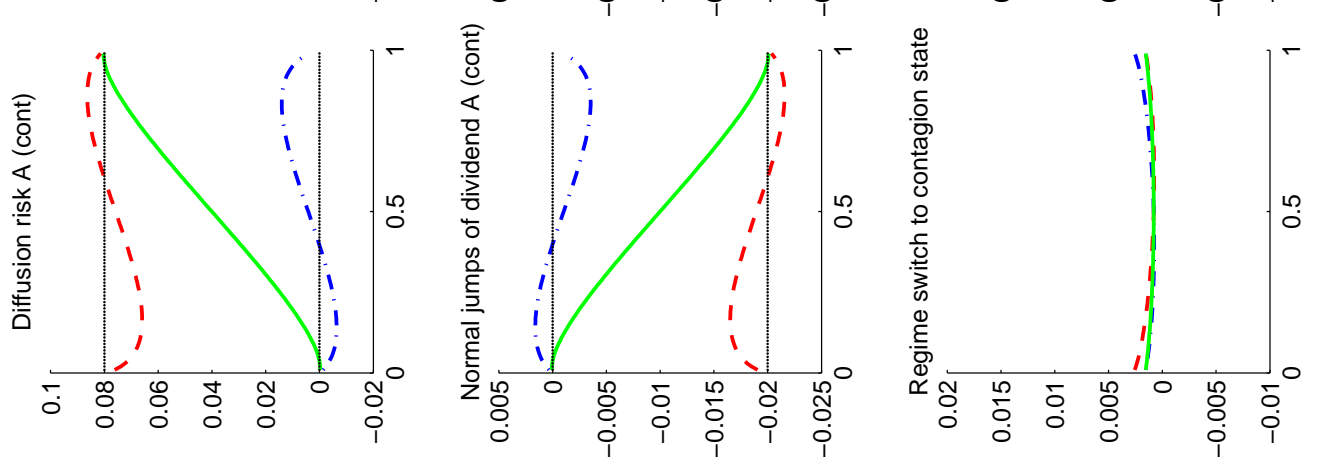

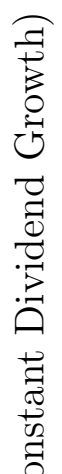
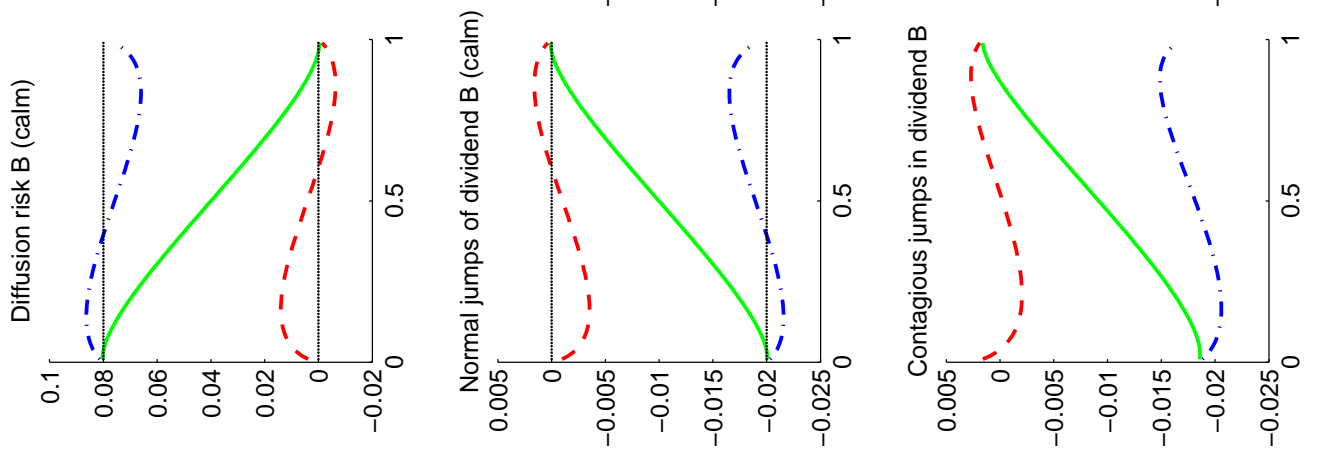

0
0
0
0
0
0
0
0
0
01
0
0
0
0
0
0
01
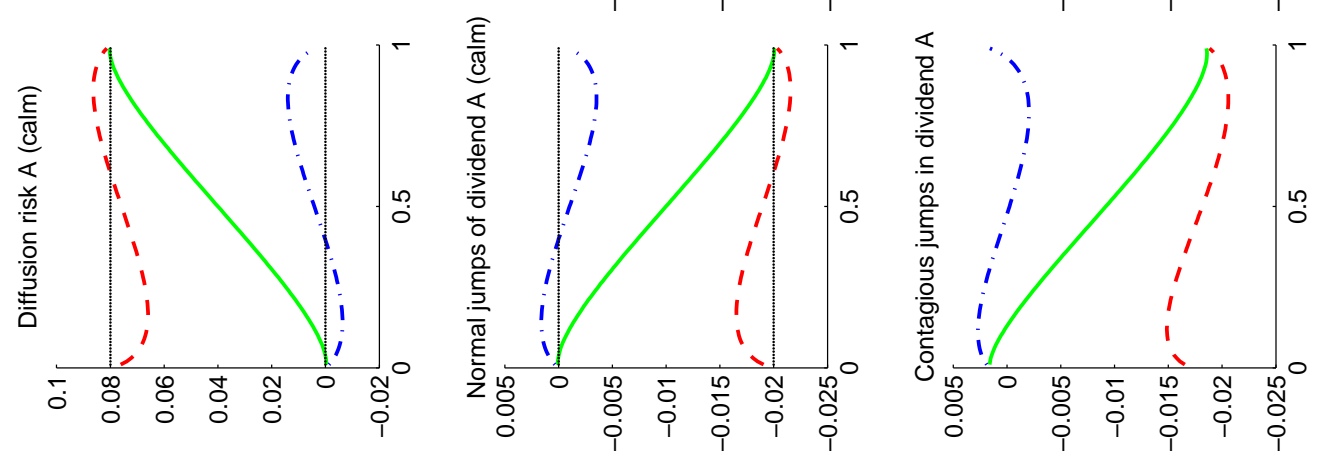

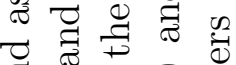

สี

$\varangle .50 .5$

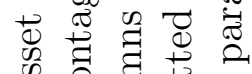

\% 잉

पै 8 ठ

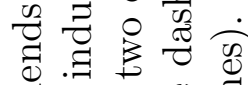

.

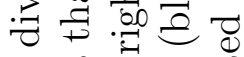

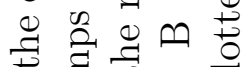

$\exists \exists+$

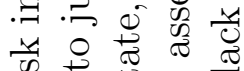

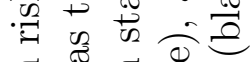

.

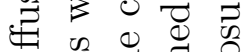

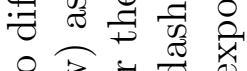

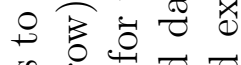

पु

월

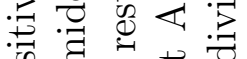

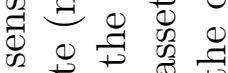

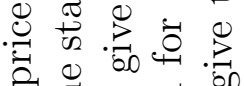

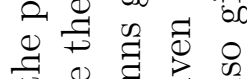

क

矛过范范

क

$\exists$ 空

舟 웡

焉焉 